\title{
Nonlinear Dynamics of Non-uniform Current-Vortex Sheets in Magnetohydrodynamic Flows
}

\author{
C. Matsuoka, K. Nishihara, T. Sano
}

\begin{tabular}{|c|l|}
\hline Citation & Journal of Nonlinear Science, 27(2): 531-572 \\
\hline Issue Date & $2017-04$ \\
\hline Type & Journal Article \\
\hline Textversion & author \\
\hline Rights & $\begin{array}{l}\text { This is a post-peer-review, pre-copyedit version of an article published in Journal } \\
\text { of Nonlinear Science. The final authenticated version is available online at: } \\
\text { https://doi.org/10.1007/s00332-016-9343-4 }\end{array}$ \\
\hline DOI & $10.1007 / \mathrm{s} 00332-016-9343-4$ \\
\hline
\end{tabular}

\author{
Self-Archiving by Author(s) \\ Placed on: Osaka City University
}

Matsuoka, C., Nishihara, K. \& Sano, T. Nonlinear Dynamics of Non-uniform Current-Vortex Sheets in Magnetohydrodynamic Flows. Journal of Nonlinear Science. 27, 531-572 (2017). 


\title{
Nonlinear dynamics of non-uniform current-vortex sheets in magnetohydrodynamic flows
}

\author{
C Matsuoka ${ }^{1}$, K. Nishihara ${ }^{2}$ and T. Sano ${ }^{2}$ \\ ${ }^{1}$ Laboratory of Applied Mathemetics, Graduate School of Engineering, \\ Osaka City University, Sugimoto-cho, Sumiyoshi, Osaka 558-8585, Japan \\ ${ }^{2}$ Institute of Laser Engineering, Osaka University, Suita, Osaka 565-0871, Japan
}

\begin{abstract}
A theoretical model is proposed to describe fully nonlinear dynamics of interfaces in twodimensional MHD flows based on an idea of non-uniform current-vortex sheet. Application of vortex sheet model to MHD flows has a crucial difficulty because of non-conservative nature of magnetic tension. However it is shown that when a magnetic field is initially parallel to an interface, the concept of vortex sheet can be extended to MHD flows (current-vortex sheet). Two-dimensional MHD flows are then described only by a one-dimensional Lagrange parameter on the sheet. It is also shown that bulk magnetic field and velocity can be calculated from their values on the sheet. The model is tested by MHD Richtmyer-Meshkov instability with sinusoidal vortex sheet strength. Two-dimensional ideal MHD simulations show that the nonlinear dynamics of a shocked interface with density stratification agrees fairly well with that for its corresponding potential flow. Numerical solutions of the model reproduce properly the results of the ideal MHD simulations, such as the roll-up of spike, exponential growth of magnetic field, and its saturation and oscillation. Nonlinear evolution of the interface is found to be determined by the Alfvén and Atwood numbers. Some of their dependence on the sheet dynamics and magnetic field amplification are discussed. It is shown by the model that the magnetic field amplification occurs locally associated with the nonlinear dynamics of the current-vortex sheet. We expect that our model can be applicable to a wide variety of MHD shear flows.
\end{abstract}

Keywords Non-uniform current-vortex sheet, Richtmyer-Meshkov instability, Alfvén number, Surface Alfvén wave, MHD interfacial instability

Mathematics Subject Classification 76W05, 76E17, 76B47, 76E30

\section{Introduction}

Motions of vortex and current sheets play an important role in various phenomena of hydrodynamic and magnetohydrodynamic (MHD) flows (Saffman, 1992, Axford and Hines, 1961, Shore, 2007). In this paper, we have developed a two-dimensional current-vortex sheet model that can describe fully-nonlinear evolutions of MHD shear flows.

For pure hydrodynamics, it is well-known that the two dimensional vortex sheet model can describe the nonlinear evolutions of shear flows, such as a transition to the global deformation 
of a vortex sheet and the appearance of new stable configurations of a spiral formation due to shear flow instability (Saffman, 1992, Godreche and Manneville, 1998), and also nonlinear interfacial instabilities such as the Kelvin-Helmholtz instability (KHI) (Krasny, 1986, 1987, Shelley, 1992), Rayleigh-Taylor instability (RTI) (Baker et al., 1982, Matsuoka and Nishihara, 2006), and Richtmyer-Meshkov instability (RMI) (Matsuoka and Nishihara, 2006, Nishihara et al., 2010). The vortex sheet equations are regularized by the vortex blob method (Krasny, 1987), and then the sheet model has succeeded to reproduce experimental results of the spiral structure (Nitsche and Krasny, 1994). The method has been applied to the nonlinear analysis of various vortical flows, which has an advantage that it can reduce spatially two-dimensional hydrodynamic equations to one-dimensional ones only for a Lagrange parameter along a sheet.

The extension of the vortex sheet model to MHD flows, which is called the current-vortex sheet (Axford, 1960), has a crucial difficulty because the magnetic tension term in the MHD Euler equation is not a conservative force. Then, Kelvin's circulation theorem, which ensures the conservation of the circulation, does not hold in MHD flows. Thus, the previous works on the theoretical analysis have been limited to either the linear stability theory (Gerwin, 1967, Chen and Hasegawa, 1974, Arshukova et al., 2002, Ilin et al., 2003, Cao et al., 2008) or the weakly nonlinear analysis (Hunter and Thoo, 2011).

However, it is found that when the magnetic field does not initially possess the normal component to the interface, the concept of vortex sheet model can be extended naturally to the currentvortex sheet model in MHD flows including the nonlinear regime. In this paper, we propose a MHD sheet model to describe the fully nonlinear motion of an interface even with non-uniform vorticity and current distribution along the interface and also density stratification. It should also be mentioned that the velocity and magnetic fields in the bulk can be derived from quantities on the sheet, if the incompressibility and the initial current free condition in the bulk are satisfied.

To demonstrate the validity of our new model of current-vortex sheets in MHD flows, we performed numerical calculations for the nonlinear evolution of MHD RMI by the sheet model as well as by ideal MHD simulations. RMI is a shock-induced inviscid interfacial instability with density stratification (Richtmyer, 1960, Meshkov, 1969, Brouillette, 2002, Nishihara et al., 2010), which is essentially driven by non-uniform velocity shear at the interface left by shocks (Fraley, 1986, Samtaney and Zabusky, 1993, 1994, Wouchuk and Nishihara, 1996, 1997). There are two sources of vorticity generation, which are the baroclinic effect and shock curvature effect (Kevkaham, 1997). Vorticity generation by the baroclinic term has been intensively discussed by Zabusky et al. (Hawley and Zabusky, 1989, Samtaney and Zabusky, 1993, 1994). However, for the linear growth of RMI, only the shock curvature effect is required as explained below (Wouchuk and Nishihara, 1996, 1997, Wouchuk et al., 2009), and the baroclinic effect is not essential. If we consider a case that a uniform incident shock passes through a corrugated interface between two fluids from light fluid to heavy one as first introduced by Richtmyer, the shock fronts of the reflected and transmitted shocks are then deformed as shown in figure 1. Fluids undergo refraction across the distorted shock fronts so that the normal component of the fluid velocity is determined from the shock Rankine-Hugoniot relation, while the tangential fluid velocity is conserved, for small amplitude of the shock ripple, as indicated by arrows in moving frames with each shock fronts in figure 1. The non-uniform velocity shear at the interface is thus left by the rippled shocks in the linear RMI. The interaction among the corrugated interface and the corrugated shock fronts then takes place through sound waves, 


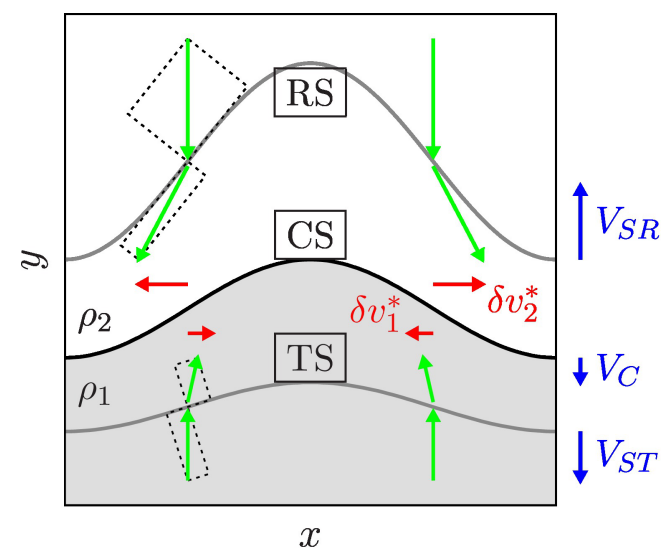

Fig. 1: A schematic diagram of Richtmyer-Meshkov instability after an incident shock pass through a corrugated contact surface (CS) between light fluid (top) and heavy fluid (bottom), where $\rho_{1}$ and $\rho_{2}$ are their mass densities after the shock interaction. Rippled transmitted shock (TS) and reflected shock (RS) propagate down ward and upward respectively. Arrows indicate perturbed fluid flow across the rippled shock surfaces in moving frames with the shocks, where normal components are determined from linearized shock jump condition, while tangential components are conserved. Here, $\delta v_{1}^{*}$ and $\delta v_{2}^{*}$ are initial transverse fluid velocities thus induced at the rippled contact surface.

as Richtmyer (Richtmyer, 1960) successfully calculated numerically, and Wouchuk and Nishihara (Wouchuk and Nishihara, 1996) gave analytical solutions of the wave equations with the form of infinite series. The initial circulation is then modified by the sound wave radiated by the rippled shocks as they go away. Through the interaction, the normal velocity at the interface reaches an asymptotic value (refer to Appendix E).

RMI is especially important in inertial fusion implosion (Ma et al., 2013, Perkins et al., 2013, Hurricane et al., 2014, Goncharov, 1999, Agliskiy et al., 2001, Ishizaki and Nishihara, 1997) and supernova explosion (Arnett et al., 1989). This instability causes the degradation of fusion yield through seeding RTI (Hurricane et al., 2014). Recently strong magnetic fields are observed in laser plasmas (Stamper et al., 1978, Daido et al., 1986, Yoneda et al., 2012, Fujioka et al., 2013) and the strong fields may affect dynamics of RMI (Cao et al., 2008, Samtaney, 2003, Sano et al., 2013, Wheatley et al., 2014, Mostert et al., 2015). It is widely known that the interstellar medium (ISM) has non-uniform structures of warm and cold neutral media (Field and Hutchins, 1968, Koyama and Inutake, 2002). RMI can take place when supernova shocks pass through ISM, which is quite common events in ISM. Supernova remnants (SNRs) are expected as a site of magnetic amplification. Recent discovery of the year-scale variability in the synchrotron X-ray emission at the downstream regions of supernova shocks suggests that the large magnetic field amplification takes place up to milligauss-order (Uchiyama et al., 2007). Moreover, the presence of a milligauss magnetic field has a critical meaning in the long standing paradigm of cosmicray proton acceleration in young SNRs (Giacalone and Jokipii, 2007, Inoue et al., 2012). We have constructed our theory inspired by this discovery of a milligauss magnetic field in SNRs and importance of the paradigm. The magnetic field amplification by vortices and the suppression of fluid instabilities by a magnetic field is a recent hot topic in MHD turbulence (Muller and Grappin, 
2005, Alexakis, 2013) as well as the inertial fusion plasmas and ISM. These studies treat the case of homogeneous plasmas, in which they report that field-line deformation by turbulent motions leads to magnetic field amplification.

Outline of this paper is as follows. In section 2, a set of fully nonlinear current-vortex sheet equations is derived, which provides mutual coupling equations between vortex sheet strength and magnetic field. In section 3, we provide the linear theory and show that the eigenmode of our model represents the surface Alfvén wave (Gerwin, 1967, Chen and Hasegawa, 1974). In section 4, we present the method to calculate the magnetic field in the whole region including the bulk based on our sheet model. Using the two-dimensional ideal MHD simulations, we show that the nonlinear evolution of the MHD RMI can be approximated by the results obtained with the initial conditions of potential flow in section 5. In section 6, we apply our current-vortex sheet model to the nonlinear evolution of various potential flows. Obtained results by our new model are compared with the corresponding ideal MHD simulations, and which shows fairly good agreements in characteristic behavior of the instability such as the roll up of spike and magnetic field amplification. Two parameters, the Alfvén and Atwood numbers are found to characterize the nonlinear evolution of the current-vortex sheet. Nonlinear dynamics of the current-vortex sheet are investigated for various conditions of the two parameters. Finally, our conclusions are summarized in section 7 .

\section{Derivation of sheet model in MHD flows}

We consider 2D inviscid and incompressible MHD flows in the Cartesian coordinate $(x, y)$. The governing equations are given by

$$
\begin{aligned}
& \frac{\partial \boldsymbol{u}}{\partial t}+(\boldsymbol{u} \cdot \nabla) \boldsymbol{u}-\frac{1}{\rho \mu}(\boldsymbol{B} \cdot \nabla) \boldsymbol{B}=-\frac{1}{\rho} \nabla\left(p+\frac{\boldsymbol{B} \cdot \boldsymbol{B}}{2 \mu}\right), \\
& \frac{\partial \boldsymbol{B}}{\partial t}=\nabla \times(\boldsymbol{u} \times \boldsymbol{B}), \\
& \nabla \cdot \boldsymbol{u}=0, \\
& \nabla \cdot \boldsymbol{B}=0, \\
& \mu \boldsymbol{j}=\nabla \times \boldsymbol{B},
\end{aligned}
$$

where $\boldsymbol{u}$ denotes the fluid velocity related with the velocity potential $\phi$ as $\boldsymbol{u}=\nabla \phi, \boldsymbol{B}$ the magnetic field, $\boldsymbol{j}$ the current density, $\rho$ the mass density, $p$ the fluid pressure, and $\mu$ the permeability (hereafter, $\mu=1$ ).

We discuss nonlinear dynamics of an interface separating two different plasmas $(i=1,2)$ with mass density $\rho_{i=1} \geq \rho_{i=2}$, which are constants (refer to Appendix E), when a non-uniform velocity shear is initially deposited on the interface. Since the interface is assumed to be a contact discontinuity, there is no flow across the interface but tangential velocity shear. The velocity shear varies along the interface. In the bulk, the irrotational potential flow is assumed, which decays exponentially away from the interface. Uniform magnetic field in each region is considered to be initially parallel to the interface. We will show that this initial value problem can be solved by introducing a non-uniform current-vortex sheet accompanied with tangential discontinuities of both the plasma velocity and the magnetic field. 
We take the curl of (2.1) and (2.2). Using the incompressible conditions (2.3) and (2.4), we obtain the evolution equations for the vorticity $\boldsymbol{\omega}=\nabla \times \boldsymbol{u}$ and the current density $\boldsymbol{j}$, respectively:

$$
\begin{aligned}
\frac{\mathrm{d} \boldsymbol{\omega}}{\mathrm{d} t} & =\frac{1}{\rho}(\boldsymbol{B} \cdot \nabla) \boldsymbol{j} \\
\frac{\mathrm{d} \boldsymbol{j}}{\mathrm{d} t} & =(\boldsymbol{u} \cdot \nabla) \boldsymbol{j}+\triangle(\boldsymbol{B} \times \boldsymbol{u}),
\end{aligned}
$$

where $\boldsymbol{j}=j \hat{\boldsymbol{e}}_{z}$ and $\boldsymbol{\omega}=\omega \hat{\boldsymbol{e}}_{z}$ ( $\hat{\boldsymbol{e}}_{z}$ : the unit vector in the $z$ direction), and $\mathrm{d} / \mathrm{d} t$ is the Lagrange derivative along the fluid motion $\mathrm{d} / \mathrm{d} t=\partial / \partial t+\boldsymbol{u} \cdot \nabla$. It should be noted that the interface is excluded in the above calculation because there exist tangential discontinuities in the magnetic and velocity fields across the interface; therefore, we cannot perform the differentiation there. [For the interface, we take the surface rotation (2.27) instead of this calculation and consider the evolution of the circulation (2.28).]

Here, we assume that the right-hand sides of equations (2.6) and (2.7) are Lipschitz continuous (when we express it roughly, the right-hand sides are possible to differentiate with respect to the dependent variables $j$ and $\omega$ and their derivatives are bounded); i.e., we exclude unphysical solutions such that they become singular in finite time when $\boldsymbol{j}$ and $\boldsymbol{\omega}$ have sufficiently smooth initial conditions. Then from the uniqueness of the solution, when $\boldsymbol{j}=0$ and $\triangle(\boldsymbol{B} \times \boldsymbol{u})=0$ are satisfied at $t=0$,

$$
\frac{\mathrm{d} \boldsymbol{\omega}}{\mathrm{d} t}=0 \quad \text { and } \quad \frac{\mathrm{d} \boldsymbol{j}}{\mathrm{d} t}=0
$$

hold for $t \geq 0$. Therefore, when the current density and vorticity satisfies the conditions $\boldsymbol{j}=0$ and $\boldsymbol{\omega}=0$ at $t=0$ in the bulk,

$$
\boldsymbol{j}(t)=0 \text { and } \boldsymbol{\omega}(t)=0
$$

for all $t>0$ in the bulk. This result guarantees that the current density and vorticity concentrate on the interface and the flow field is only determined by the dynamics of the interface. These current and vorticity free conditions in the bulk are the starting point of our sheet model.

In the following analysis, we adopt the sheet model (Baker et al., 1982, Matsuoka et al., 2003, Matsuoka and Nishihara, 2006). We consider the dynamics of an interface separating two different plasmas. In our current-vortex sheet model, we consider only potential flow in each region. However, if the potential flow in each region has different tangential velocity across the interface separating two plasmas, the vorticity (the velocity shear) and therefore, the circulation is induced at the interface. We solve nonlinear time evolution of the circulation at the interface together with the interfacial dynamics induced. Then we parameterize points on the interface as

$$
\boldsymbol{X}(\theta, t)=[X(\theta, t), Y(\theta, t)]
$$

and its velocity as

$$
\boldsymbol{u}^{+}(\theta, t) \equiv\left(\frac{\mathrm{d} X(\theta, t)}{\mathrm{d} t}, \frac{\mathrm{d} Y(\theta, t)}{\mathrm{d} t}\right)
$$

using a Lagrangian marker $\theta$, where

$$
\frac{\mathrm{d}}{\mathrm{d} t}=\frac{\partial}{\partial t}+\boldsymbol{u}^{+} \cdot \nabla
$$

is the Lagrangian derivative in the frame moving with the interface. By introducing this model, all physical quantities in the governing equations $(2.1)-(2.5)$ described by $(x, y ; t)$ are reduced to 
the ones described by $(\theta ; t)$; i.e., 2D problems are reduced to $1 \mathrm{D}$ problems. Hereafter, we omit the parameter $\theta$. The interface velocity can be chosen as

$$
\boldsymbol{u}^{+}=\boldsymbol{q}+\frac{\tilde{\alpha}}{2} \gamma
$$

so that it satisfies the continuity of the normal velocity at the interface. Here,

$$
\boldsymbol{q} \equiv(U, V)=\frac{\boldsymbol{u}_{1, i n t}+\boldsymbol{u}_{2, i n t}}{2}
$$

is the average velocity of plasma 1 and 2 at the point, and

$$
\gamma=\boldsymbol{u}_{1, i n t}-\boldsymbol{u}_{2, i n t},
$$

where $|\gamma|=\gamma$ denotes the vortex sheet strength derived from the circulation

$$
\Gamma \equiv \phi_{1, i n t}-\phi_{2, i n t}
$$

at the interface as $\gamma=\boldsymbol{t} \partial \Gamma / \partial s$, where the subscript $i$, int indicates its value of $i$-plasma at the Lagrangian point of the interface, and $s$ and $\boldsymbol{t}$ are length and the unit tangent of the interface, respectively. The artificial parameter (weighting factor) $\tilde{\alpha}$ in (2.10) is a function of the Atwood number

$$
A \equiv \frac{\rho_{2}-\rho_{1}}{\rho_{1}+\rho_{2}}
$$

( $A \leq 0$ in this paper) and $\tilde{\alpha} \neq 0$ for $A \neq 0$ (Baker et al., 1982, Pullin, 1982). The physical quantities $\boldsymbol{q}, \boldsymbol{\gamma}$, and $\Gamma$ are defined on the interface as $(\theta ; t)$.

Then the sheet velocity $\mathrm{d} \boldsymbol{X} / \mathrm{d} t$ is written as (Baker et al., 1982, Matsuoka and Nishihara, 2006)

$$
\frac{\mathrm{d} X}{\mathrm{~d} t}=U+\frac{\tilde{\alpha} X_{\theta}}{2 s_{\theta}} \gamma, \quad \frac{\mathrm{d} Y}{\mathrm{~d} t}=V+\frac{\tilde{\alpha} Y_{\theta}}{2 s_{\theta}} \gamma,
$$

which is related to the vortex-induced velocity described by the Biot-Savart law (Birkhoff-Rott equation) (Birkhoff, 1962, Rott, 1956):

$$
q^{*}=U-i V=\frac{1}{2 \pi i} \mathrm{P} . \mathrm{V} \cdot \int_{-\infty}^{\infty} \frac{\gamma\left(\theta^{\prime}\right) s_{\theta}\left(\theta^{\prime}\right) d \theta^{\prime}}{Z(\theta)-Z\left(\theta^{\prime}\right)}
$$

where the subscript $\theta$ denotes differentiation with respect to $\theta, Z=X+i Y, s_{\theta}=\sqrt{X_{\theta}^{2}+Y_{\theta}^{2}}$, and P.V. denotes the principal integral. Using $\boldsymbol{q}$ and $\boldsymbol{\gamma}$, the velocities $\boldsymbol{u}_{1, \text { int }}$ and $\boldsymbol{u}_{2, \text { int }}$ are written as

$$
\boldsymbol{u}_{1, i n t}=\boldsymbol{q}+\frac{\gamma}{2} \quad \text { and } \quad \boldsymbol{u}_{2, i n t}=\boldsymbol{q}-\frac{\gamma}{2}
$$

which satisfy the continuity of the normal velocity at the interface. For the magnetic field such that it is initially parallel to the interface, the following statement holds.

Proposition The magnetic field that satisfies $\boldsymbol{B}_{i, \text { int }}=B_{i, \text { int }} \boldsymbol{t}$ at $t=0$ does not have the normal component when $t>0$.

Proof. From the equation $\mathrm{d}(\boldsymbol{n} \cdot \boldsymbol{n}) / \mathrm{d} t=0$ for the unit normal $\boldsymbol{n}$ of the interface, we see that $\mathrm{d} \boldsymbol{n} / \mathrm{d} t=c \boldsymbol{t}$, where $c$ is a constant. Using $\mathrm{d}(\boldsymbol{n} \cdot \boldsymbol{t}) / \mathrm{d} t=0$, we have

$$
\frac{\mathrm{d} \boldsymbol{n}}{\mathrm{d} t} \cdot \boldsymbol{t}=c=-\boldsymbol{n} \cdot \frac{\mathrm{d} \boldsymbol{t}}{\mathrm{d} t} .
$$


The relation

$$
\frac{\mathrm{d} \boldsymbol{t}}{\mathrm{d} t}=\frac{\mathrm{d}}{\mathrm{d} t}\left(\frac{\partial \boldsymbol{X}}{\partial s}\right)=\frac{\partial}{\partial s}\left(\frac{\mathrm{d} \boldsymbol{X}}{\mathrm{d} t}\right)=\frac{1}{s_{\theta}} \frac{\mathrm{d} \boldsymbol{X}_{\theta}}{\mathrm{d} t}
$$

yields

$$
\frac{\mathrm{d} \boldsymbol{n}}{\mathrm{d} t} \cdot \boldsymbol{t}=c=-\frac{1}{s_{\theta}}\left(\frac{\mathrm{d} \boldsymbol{X}_{\theta}}{\mathrm{d} t} \cdot \boldsymbol{n}\right) .
$$

From equations (2.14) and (2.15), we obtain

$$
\frac{\mathrm{d} t}{\mathrm{~d} t}=-c \boldsymbol{n}=\frac{\boldsymbol{n}}{s_{\theta}}\left(\frac{\mathrm{d} \boldsymbol{X}_{\theta}}{\mathrm{d} t} \cdot \boldsymbol{n}\right), \quad \frac{\mathrm{d} \boldsymbol{n}}{\mathrm{d} t}=c \boldsymbol{t}=-\frac{\boldsymbol{t}}{s_{\theta}}\left(\frac{\mathrm{d} \boldsymbol{X}_{\theta}}{\mathrm{d} t} \cdot \boldsymbol{n}\right) .
$$

The induction equation (2.2) and the relation (2.16) lead to

$$
\begin{aligned}
& \frac{\mathrm{d}}{\mathrm{d} t_{i}}\left(\boldsymbol{B}_{i, i n t} \cdot \boldsymbol{n}\right)=\frac{\mathrm{d} \boldsymbol{B}_{i, i n t}}{\mathrm{~d} t_{i}} \cdot \boldsymbol{n}+\frac{\mathrm{d} \boldsymbol{n}}{\mathrm{d} t_{i}} \cdot \boldsymbol{B}_{i, i n t} \\
= & {\left[\left(\boldsymbol{B}_{i, i n t} \cdot \nabla\right) \boldsymbol{u}_{i, i n t}\right] \cdot \boldsymbol{n}+\boldsymbol{B}_{i, i n t} \cdot\left[\frac{\mathrm{d}}{\mathrm{d} t}+\left(\boldsymbol{u}_{i, i n t}-\boldsymbol{u}^{+}\right) \cdot \nabla\right] \boldsymbol{n} } \\
= & \frac{B_{i, i n t}^{t}}{s_{\theta}}\left(\boldsymbol{q}_{\theta} \pm \frac{1}{2} \gamma_{\theta}\right) \cdot \boldsymbol{n}-\boldsymbol{B}_{i, i n t} \cdot\left[\frac{\boldsymbol{t}}{s_{\theta}}\left(\frac{\mathrm{d} \boldsymbol{X}_{\theta}}{\mathrm{d} t} \cdot \boldsymbol{n}\right)+\frac{1 \mp \tilde{\alpha}}{2} \gamma \kappa\right] \\
= & \pm \frac{(1 \mp \tilde{\alpha}) B_{i, i n t}^{t}}{2 s_{\theta}}\left(\gamma_{\theta} \cdot \boldsymbol{n}\right) \mp \frac{(1 \mp \tilde{\alpha}) B_{i, i n t}^{t}}{2} \gamma \kappa=0,
\end{aligned}
$$

where the upper (lower) sign denotes $i=1(i=2), \kappa$ the curvature of the interface,

$$
\frac{\mathrm{d}}{\mathrm{d} t_{i}}=\frac{\partial}{\partial t}+\boldsymbol{u}_{i, i n t} \cdot \nabla
$$

and we used the relation

$$
\gamma_{\theta}=s_{\theta} \frac{\partial \gamma}{\partial s}, \quad \frac{\partial \gamma}{\partial s} \cdot \boldsymbol{n}=\frac{\partial}{\partial s}(\gamma \boldsymbol{t}) \cdot \boldsymbol{n}=\gamma \kappa,
$$

where the Frenet-Serret formula in the plane curves is used for deriving the second equation of equation (2.19).

Using (2.17), we obtain the relation

$$
\frac{\mathrm{d}}{\mathrm{d} t}\left(\boldsymbol{B}_{i, i n t} \cdot \boldsymbol{n}\right)=\left[\frac{\mathrm{d}}{\mathrm{d} t_{i}}+\left(\boldsymbol{u}^{+}-\boldsymbol{u}_{i, i n t}\right) \cdot \nabla\right] \boldsymbol{B}_{i, i n t} \cdot \boldsymbol{n}=\frac{\tilde{\alpha} \mp 1}{2 s_{\theta}} \gamma \frac{\partial B_{i, i n t}^{n}}{\partial \theta}=0
$$

for the case that the condition $B_{i, i n t}^{n}=\boldsymbol{B}_{i, i n t} \cdot \boldsymbol{n}=0$ is satisfied at $t=0$. It follows from this equation that no normal magnetic field across the interface exists, if the field is initially parallel to the interface; i.e.,

$$
\boldsymbol{B}_{i, i n t} \cdot \boldsymbol{n}=0
$$

holds for all $t \geq 0$. Thus, the statement is proved.

This proposition indicates that the "frozen-in" condition holds even for the interface that moves with the velocity $\boldsymbol{u}^{+}$. From this proposition, we see that the dynamics of a current-vortex sheet is reduced to the motion of the tangential direction of that. Hereafter, we omit the subscript int.

Now we derive the evolution equation for the tangential magnetic field $B_{i}^{t} \equiv \boldsymbol{B}_{i} \cdot \boldsymbol{t}$. From $(2.16)$ and (2.18), we obtain

$$
\begin{aligned}
& \frac{\mathrm{d} B_{i}^{t}}{\mathrm{~d} t}=\frac{\mathrm{d}}{\mathrm{d} t}\left(\boldsymbol{B}_{i} \cdot \boldsymbol{t}\right)=\frac{\mathrm{d} \boldsymbol{B}_{i}}{\mathrm{~d} t} \cdot \boldsymbol{t}+\boldsymbol{B}_{i} \cdot \frac{\mathrm{d} \boldsymbol{t}}{\mathrm{d} t}=\frac{\mathrm{d} \boldsymbol{B}_{i}}{\mathrm{~d} t} \cdot \boldsymbol{t} \\
= & {\left[\frac{\mathrm{d}}{\mathrm{d} t_{i}}+\left(\boldsymbol{u}^{+}-\boldsymbol{u}_{i}\right) \cdot \nabla\right] \boldsymbol{B}_{i} \cdot \boldsymbol{t} } \\
= & \frac{\mathrm{d} \boldsymbol{B}_{i}}{\mathrm{~d} t_{i}} \cdot \boldsymbol{t}+\left(\boldsymbol{u}^{+}-\boldsymbol{u}_{i}\right) \cdot \nabla\left(\boldsymbol{B}_{i} \cdot \boldsymbol{t}\right),
\end{aligned}
$$


where we used the relation $\mathrm{d} \boldsymbol{t} / \mathrm{d} t_{i} \propto \boldsymbol{n}$. Using the divergence conditions (2.3) and (2.4), the induction equation (2.2) can be rewritten as

$$
\frac{\mathrm{d} \boldsymbol{B}_{i}}{\mathrm{~d} t_{i}}=\left(\boldsymbol{B}_{i} \cdot \nabla\right) \boldsymbol{u}_{i}
$$

in each fluid $i$. From (2.22) and (2.23), we can derive the evolution equation for the tangential magnetic field $B_{i}^{t}$ as

$$
\frac{\mathrm{d} B_{i}^{t}}{\mathrm{~d} t}=\frac{\tilde{\alpha} \mp 1}{2 s_{\theta}} \gamma B_{i, \theta}^{t}+\frac{B_{i}^{t}}{s_{\theta}}\left(q_{\theta}^{t} \pm \frac{\gamma_{\theta}}{2}\right),
$$

where $B_{i, \theta}^{t}=\partial B_{i}^{t} / \partial \theta, q_{\theta}^{t}=\partial(\boldsymbol{q} \cdot \boldsymbol{t}) / \partial \theta$, and the upper (lower) sign corresponds to $i=1(i=2)$.

Now we derive the evolution equation for the vortex sheet strength $\gamma$. As shown in (2.9), the current density $\boldsymbol{j}$ in the bulk satisfies $\boldsymbol{j}=0$. Therefore, the Lorentz force term $\boldsymbol{j} \times \boldsymbol{B}=$ $-\nabla(\boldsymbol{B} \cdot \boldsymbol{B} / 2)+(\boldsymbol{B} \cdot \nabla) \boldsymbol{B}$ in the Euler equation (2.1) is zero in the bulk. This enables us to replace the magnetic tension (the non-conservative force) with the magnetic pressure (the conservative force) in each fluid $i$; i.e., the relation

$$
\left(\boldsymbol{B}_{i} \cdot \nabla\right) \boldsymbol{B}_{i}=\nabla\left(\frac{\boldsymbol{B}_{i} \cdot \boldsymbol{B}_{i}}{2}\right)
$$

holds.

Imposing a condition that the total pressure (normal stress) $P=p+\boldsymbol{B} \cdot \boldsymbol{B} / 2$ is identical across the interface:

$$
p_{1}+\frac{\boldsymbol{B}_{1} \cdot \boldsymbol{B}_{1}}{2}=p_{2}+\frac{\boldsymbol{B}_{2} \cdot \boldsymbol{B}_{2}}{2},
$$

we integrate the Euler equation (2.1) along a line surrounding the interface $\oint \cdot \mathrm{d} \boldsymbol{x}$ (for this integration method, e.g., see Saffman, 1992). Then, using the conditions (2.25) and (2.26), and taking the limit of thickness to be zero for the interface, we obtain the Bernoulli equation for the velocity potential $\phi_{i}$ :

$$
\begin{aligned}
& (1-A)\left[\frac{\partial \phi_{1}}{\partial t}+\frac{1}{2}\left(\nabla \phi_{1}\right)^{2}\right]-(1+A)\left[\frac{\partial \phi_{2}}{\partial t}+\frac{1}{2}\left(\nabla \phi_{2}\right)^{2}\right] \\
= & \frac{1}{\rho_{1}+\rho_{2}}\left(\boldsymbol{B}_{1} \cdot \boldsymbol{B}_{1}-\boldsymbol{B}_{2} \cdot \boldsymbol{B}_{2}\right) .
\end{aligned}
$$

Using the relation (2.11), the Bernoulli equation (2.27) is rewritten as the following evolution equation for the circulation $\Gamma$ :

$$
\frac{\mathrm{d} \Gamma}{\mathrm{d} t}=2 A \frac{\mathrm{d} \Phi}{\mathrm{d} t}-A \boldsymbol{q} \cdot \boldsymbol{q}+\frac{A+2 \tilde{\alpha}}{4} \boldsymbol{\gamma} \cdot \boldsymbol{\gamma}-\tilde{\alpha} A \boldsymbol{\gamma} \cdot \boldsymbol{q}+\frac{2}{\rho_{1}+\rho_{2}}\langle\boldsymbol{B}\rangle \cdot \boldsymbol{j}_{s},
$$

where $\Phi=\left(\phi_{1}+\phi_{2}\right) / 2,\langle\boldsymbol{B}\rangle=\left(\boldsymbol{B}_{1}+\boldsymbol{B}_{2}\right) / 2$,

$$
\boldsymbol{j}_{s}=\boldsymbol{B}_{1}-\boldsymbol{B}_{2}
$$

denotes the surface current density, and $j_{s}=\boldsymbol{j}_{s} \cdot \boldsymbol{t}$ provides the current sheet strength. For the relation between the current density $\boldsymbol{j}$ and the surface current density $\boldsymbol{j}_{s}$, refer to Appendix A. In deriving (2.28), we also use the fact that the vorticity $\boldsymbol{\omega}=0$ in the bulk. It should be noted that $\mathrm{d} \Gamma / \mathrm{d} t \neq 0$ in MHD flows even for $A=0$, due to the existence of the last term in (2.28) associated 
with the magnetic tension; i.e., the Kelvin's circulation law $\Gamma=$ constant does not hold in this system even for the homogeneous fluid.

Differentiating (2.28) with respect to $\theta$, we obtain the following Fredholm integral equation of the second kind:

$$
\begin{aligned}
\frac{\mathrm{d} \gamma}{\mathrm{d} t} & =\frac{2 A}{s_{\theta}}\left(X_{\theta} \frac{\mathrm{d} U}{\mathrm{~d} t}+Y_{\theta} \frac{\mathrm{d} V}{\mathrm{~d} t}\right)-\frac{(1+\tilde{\alpha} A) \gamma}{s_{\theta}^{2}}\left(X_{\theta} U_{\theta}+Y_{\theta} V_{\theta}\right) \\
& +\frac{A+\tilde{\alpha}}{4 s_{\theta}}\left(\gamma^{2}\right)_{\theta}+\frac{2}{s_{\theta}\left(\rho_{1}+\rho_{2}\right)}\left(\langle\boldsymbol{B}\rangle \cdot \boldsymbol{j}_{s}\right)_{\theta} .
\end{aligned}
$$

By solving (2.12), (2.13), (2.24), and (2.29) simultaneously, we can determine the fully nonlinear motion of a non-uniform current-vortex sheet. We would like to mention that the magnetic field affects the temporal evolution of local vortex sheet strength through equation (2.29), while the vortex sheet strength determines time variation of the magnetic field through equation (2.24), namely these equations provide the mutual coupling between the vortex sheet strength and the magnetic field in MHD shear flows.

\section{Linear analysis}

Before discussing the fully nonlinear dynamics of a non-uniform current-vortex sheet, we show that a linear eigenmode of the system represents the surface Alfvén wave (Gerwin, 1967, Chen and Hasegawa, 1974) with density stratification. For the linear analysis, we denote the interface as $y=\eta(x, t)$ and use the following kinematic boundary condition instead of solving the integral equation of the Biot-Savart law (2.13):

$$
\frac{\partial \eta}{\partial t}-\frac{\partial \phi_{i}}{\partial y}=\frac{\partial \phi_{i}}{\partial x} \frac{\partial \eta}{\partial x} \quad(i=1,2)
$$

We introduce a small sinusoidal perturbation of the interface as $k \tilde{\eta} \ll 1$ and the magnetic field $\left|\tilde{\boldsymbol{B}}_{i} / \boldsymbol{B}_{0}\right| \ll 1$, where $k$ is the wave number, $\boldsymbol{B}_{0}$ is a uniform magnetic field in $x$-direction $\boldsymbol{B}_{0}=$ $B_{0} \hat{\boldsymbol{e}_{x}}$, in which $B_{0}$ is a constant and $\hat{\boldsymbol{e}_{x}}$ is the unit vector in the $x$-direction. The velocity potential has only a small perturbation of $\tilde{\phi}_{i}$, where the the tilde denotes the first-order perturbations. Substituting these quantities into equations (2.3), (2.4), (2.23), (2.27), and (3.1), we obtain the linearized equations as

$$
\begin{aligned}
& \rho_{1} \frac{\partial \tilde{\phi}_{1}}{\partial t}-\rho_{2} \frac{\partial \tilde{\phi}_{2}}{\partial t}=\boldsymbol{B}_{0} \cdot\left(\tilde{\boldsymbol{B}}_{1}-\tilde{\boldsymbol{B}}_{2}\right), \\
& \frac{\partial \tilde{\boldsymbol{B}}_{i}}{\partial t}=\left(\boldsymbol{B}_{0} \cdot \nabla\right) \tilde{\boldsymbol{u}}_{i} \quad(i=1,2), \\
& \tilde{\boldsymbol{u}}_{i}=\nabla \tilde{\phi}_{i} \\
& \frac{\partial \tilde{\eta}}{\partial t}=\frac{\partial \tilde{\phi}_{i}}{\partial y}, \\
& \nabla \cdot \tilde{\boldsymbol{u}}_{i}=0, \quad \nabla \cdot \tilde{\boldsymbol{B}}_{i}=0 .
\end{aligned}
$$

As the standing wave solution of equation (3.2), we set, for example,

$$
\begin{aligned}
& \tilde{\eta}=\delta \eta \operatorname{Re}\left(\mathrm{e}^{i\left(k x-\omega t+\varphi_{+}\right)}+\mathrm{e}^{-i\left(k x+\omega t+\varphi_{-}\right)}\right), \\
& \tilde{\phi}_{1}=\delta \phi_{1} \operatorname{Re}\left(\mathrm{e}^{i\left(k x-\omega t+\varphi_{+}\right)}+\mathrm{e}^{-i\left(k x+\omega t+\varphi_{-}\right)}\right) \mathrm{e}^{k y} \quad(y<0), \\
& \tilde{\phi}_{2}=\delta \phi_{2} \operatorname{Re}\left(\mathrm{e}^{i\left(k x-\omega t+\varphi_{+}\right)}+\mathrm{e}^{-i\left(k x+\omega t+\varphi_{-}\right)}\right) \mathrm{e}^{-k y} \quad(y>0),
\end{aligned}
$$


where $\omega=\omega(k)$ is the frequency, Re denotes the real part and $\delta \eta, \delta \phi_{i}$, and $\varphi_{ \pm}$are constants. Substituting these standing wave solutions into equation (3.2), we obtain the following dispersion relation,

$$
\omega^{2}=k^{2} v_{a 0}^{2}
$$

where

$$
v_{a 0}^{2}=\frac{2 B_{0}^{2}}{\rho_{1}+\rho_{2}}
$$

is the Alfvén velocity defined by the average mass density in region 1 and 2. It should be noted that if the magnetic field in two regions is not the same, $2 B_{0}^{2}$ in equation (3.5) should be replaced by $B_{10}^{2}+B_{20}^{2}$. Namely the linear system given by equations (3.2) presents the surface Alfvén wave (Gerwin, 1967, Chen and Hasegawa, 1974) with density stratification. Cao's solution includes this eigenmode (Cao et al., 2008).

For a standing wave solution, the eigenmode satisfy the following relations

$$
\begin{aligned}
& \tilde{\phi}_{1}=\frac{\delta u}{k} \cos k x \cos (\omega t+\varphi) \mathrm{e}^{k y} \quad(y<0), \\
& \tilde{\phi}_{2}=-\frac{\delta u}{k} \cos k x \cos (\omega t+\varphi) \mathrm{e}^{-k y} \quad(y>0) \\
& \tilde{\boldsymbol{u}}_{1}=\delta u(-\sin k x, \cos k x) \cos (\omega t+\varphi) \mathrm{e}^{k y} \quad(y<0), \\
& \tilde{\boldsymbol{u}}_{2}=\delta u(\sin k x, \cos k x) \cos (\omega t+\varphi) \mathrm{e}^{-k y} \quad(y>0) \\
& \tilde{\eta}=\frac{\delta u}{k v_{a 0}} \cos k x \sin (\omega t+\varphi) \\
& \tilde{\boldsymbol{B}}_{1}=-\frac{B_{0} \delta u}{v_{a 0}}(\cos k x, \sin k x) \sin (\omega t+\varphi) \mathrm{e}^{k y} \quad(y<0), \\
& \tilde{\boldsymbol{B}}_{2}=\frac{B_{0} \delta u}{v_{a 0}}(\cos k x,-\sin k x) \sin (\omega t+\varphi) \mathrm{e}^{-k y} \quad(y>0),
\end{aligned}
$$

where $\delta u$ and $\varphi$ are constants. From the above eigenmode, the vortex sheet strength $\tilde{\gamma}$ and the surface current density $\tilde{\boldsymbol{j}}_{s}$ are given as

$$
\begin{array}{r}
\tilde{\boldsymbol{\gamma}}=-2 \delta u(\sin k x \cos (\omega t+\varphi), 0), \\
\tilde{\boldsymbol{j}}_{s}=-2 \frac{B_{0} \delta u}{v_{a 0}}(\cos k x \sin (\omega t+\varphi), 0) .
\end{array}
$$

The pure linear RMI solution is reproduced by $\tilde{\eta} \sim t \cos x$ (Wouchuk and Nishihara, 1997, Matsuoka et al., 2003) when $\omega t=k v_{\text {lin }}\left(v_{a 0} / v_{\text {lin }}\right) t=k v_{a 0} t \ll 1$. This solution coincides with the one obtained by Cao et al. (Cao et al., 2008) [equation (25) in this reference]. Linear solution also gives rise to KHI for a uniform initial sheet strength $\gamma$.

\section{Magnetic field in bulk}

In this section, we present the method to calculate the magnetic field in the whole region including the bulk under the boundary condition (2.21), by using the boundary value of the magnetic field obtained from the sheet model. In the present study, we consider a multivalued interface with the non-uniformly distributed surface current on the interface. The current-vortex sheet model itself provides only the magnetic field along the interface in each region. For the bulk magnetic field, the 
solenoidal part calculated from the surface current using the Biot-Savart equation [refer to equation (4.5)] is not enough to satisfy the boundary condition (2.21) for the interface with multivaluedness and a non-uniform current. Since $\nabla \cdot \boldsymbol{B}=0$ allows adding an irrotational potential field such that it satisfies the Laplace equation, we can set the magnetic field as

$$
\boldsymbol{B}=\boldsymbol{B}_{s}+\nabla \chi
$$

where $\boldsymbol{B}_{s}$ denotes a solenoidal vector field and $\chi$ is a irrotational potential field that satisfies $\triangle \chi=0$. Without this irrotational part, we cannot construct the bulk magnetic field that satisfies the boundary condition of no normal magnetic field at the interface. For the representation (4.1), the following theorem holds.

Theorem There exist two different solenoidal vectors $\boldsymbol{B}_{i s}$ and the irrotational potential fields $\chi_{i}(i=1,2)$, and the magnetic field $\boldsymbol{B}$ is represented by

$$
\boldsymbol{B}(\boldsymbol{x})= \begin{cases}\boldsymbol{B}_{1 s}+\nabla \chi_{1} & \left(\boldsymbol{x} \in D_{1}\right) \\ \boldsymbol{B}_{2 s}+\nabla \chi_{2} & \left(\boldsymbol{x} \in D_{2}\right) .\end{cases}
$$

Proof. From the boundary condition $(2.21), \nabla \chi \cdot \boldsymbol{n}=-\boldsymbol{B}_{s} \cdot \boldsymbol{n}$ holds. Generally, $\boldsymbol{B}_{s}(\boldsymbol{x})$ is not constant and $\boldsymbol{B}_{s}(\boldsymbol{x}) \cdot \boldsymbol{n} \neq 0$ at the interface; therefore, $\nabla \chi(\boldsymbol{x})$ is not constant at the interface and $\nabla \chi(\boldsymbol{x}) \cdot \boldsymbol{n} \neq 0$ holds. Now we decompose the whole region $D$ as $D=D_{1}+D_{2}+\partial D$, where $D_{i}$ and $\partial D$ denote the region $i(i=1,2)$ and the boundary (interface), respectively. The irrotational field $\chi$ is the solution to the following (potential) problem:

$$
\begin{aligned}
\Delta \chi & =0 \text { in } D, \quad \chi \rightarrow B_{0} x \text { as } y \rightarrow \pm \infty, \\
\nabla \chi(\boldsymbol{x}) \cdot \boldsymbol{n} & \neq 0 \text { on } \partial D .
\end{aligned}
$$

Now we introduce a harmonic function $\mu$ that is conjugate to $\chi$. Then $f=\chi+i \mu$ denotes a regular function (a complex potential) and its derivative (the complex velocity)

$$
w^{*} \equiv \frac{d f}{d z}=\frac{\partial \chi}{\partial x}-i \frac{\partial \chi}{\partial y}, \quad w=\frac{\partial \chi}{\partial x}+i \frac{\partial \chi}{\partial y},
$$

is also a regular function. The function $w$ is obviously bounded $(|w(z)|<M, \forall z \in \mathbb{C} ; M$ : a constant). From the Liouville's theorem in the complex analysis, a function that is bounded and regular in the whole plane must be constant. This is contradictory to the condition that $\nabla \chi$; the vector form of $w$, is not constant on $\partial D$. Therefore, the function $w=w(z)$ is divided into two regular functions across the interface:

$$
w= \begin{cases}w_{1} & \left(z \in D_{1}\right) \\ w_{2} & \left(z \in D_{2}\right)\end{cases}
$$

Accordingly, two different $\chi$ exist:

$$
\chi= \begin{cases}\chi_{1} & \left(z \in D_{1}\right) \\ \chi_{2} & \left(z \in D_{2}\right)\end{cases}
$$

This indicates that two different representations for the magnetic field $\boldsymbol{B}$ exist in the current problem; i.e., the magnetic field $\boldsymbol{B}$ in the upper (lower) half-plane is different from the one in the lower (upper) half-plane. Thus, the statement of the theorem is proved. 


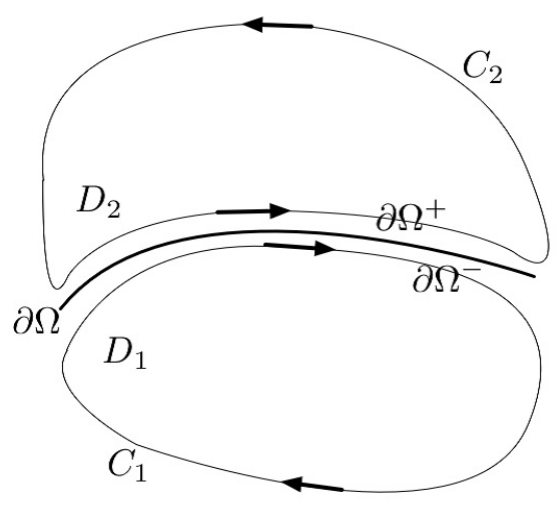

Fig. 2: Contours $C_{1}$ and $C_{2}$, where $C_{1}\left(C_{2}\right)$ rotates $D_{1}\left(D_{2}\right)$ clockwise (counterclockwise).

It should be noted that the representation of the magnetic field exists two ways but $\boldsymbol{B}$ itself is only one in the whole plane.

The boundary value of the potential field $\nabla \chi_{i} \equiv \nabla \chi_{i, i n t}$ is given by the following relation:

$$
\nabla \chi_{i, i n t}=B_{i}^{t} \boldsymbol{t}-\boldsymbol{B}_{i s, i n t}
$$

in which the subscript int denotes its boundary value. The solenoidal part $\boldsymbol{B}_{i s}=\left(B_{i s, x}, B_{i s, y}\right)$ including its boundary value $\boldsymbol{B}_{i s, i n t}$ is given with its complex form as $[\boldsymbol{x}=(x, y) \rightarrow z=x+i y]$

$$
\begin{aligned}
& B_{1 s}^{*}(z)=B_{1 s, x}-i B_{1 s, y}=\frac{1}{2 \pi i} \mathrm{P} . \mathrm{V} \cdot \int_{-\infty}^{\infty} \frac{B_{1}^{t}\left(\theta^{\prime}\right) s_{\theta}\left(\theta^{\prime}\right) d \theta^{\prime}}{z-Z\left(\theta^{\prime}\right)} \quad\left(z \in D_{1}+\partial D^{-}\right), \\
& B_{2 s}^{*}(z)=B_{2 s, x}-i B_{2 s, y}=-\frac{1}{2 \pi i} \mathrm{P} . \mathrm{V} \cdot \int_{-\infty}^{\infty} \frac{B_{2}^{t}\left(\theta^{\prime}\right) s_{\theta}\left(\theta^{\prime}\right) d \theta^{\prime}}{z-Z\left(\theta^{\prime}\right)} \quad\left(z \in D_{2}+\partial D^{+}\right)
\end{aligned}
$$

where $\partial D^{+}\left(\partial D^{-}\right)$denotes the upper (lower) side of the interface. The periodic form with respect to $x$ of this equation gives

$$
\begin{aligned}
& B_{i s, x}(x, y)=\mp \frac{1}{4 \pi} \mathrm{P} . \mathrm{V} \cdot \int_{-\pi}^{\pi} \frac{\sinh \left(y-Y\left(\theta^{\prime}\right)\right) B_{i}^{t}\left(\theta^{\prime}\right) s_{\theta}\left(\theta^{\prime}\right) d \theta^{\prime}}{\cosh \left(y-Y\left(\theta^{\prime}\right)\right)-\cos \left(x-X\left(\theta^{\prime}\right)\right)} \\
& B_{i s, y}(x, y)= \pm \frac{1}{4 \pi} \mathrm{P} . \mathrm{V} \cdot \int_{-\pi}^{\pi} \frac{\sin \left(x-X\left(\theta^{\prime}\right)\right) B_{i}^{t}\left(\theta^{\prime}\right) s_{\theta}\left(\theta^{\prime}\right) d \theta^{\prime}}{\cosh \left(y-Y\left(\theta^{\prime}\right)\right)-\cos \left(x-X\left(\theta^{\prime}\right)\right)}
\end{aligned}
$$

where the upper (lower) sign on the right-hand side corresponds to $i=1(i=2)$.

Taking into account the initial homogeneous magnetic field $\boldsymbol{B}_{0}$, the boundary value $\nabla \chi_{i, \text { int }}=$ $\left(\partial \chi_{i, i n t} / \partial x, \partial \chi_{i, i n t} / \partial y\right)$ is given as

$$
\begin{array}{lr}
\frac{\partial \chi_{i, i n t}}{\partial x}= & B_{i}^{t} t_{x}-B_{i s, i n t, x}-B_{0} \\
\frac{\partial \chi_{i, i n t}}{\partial y}= & B_{i}^{t} t_{y}-B_{i s, i n t, y}
\end{array}
$$

where we set $\boldsymbol{t}=\left(t_{x}, t_{y}\right)$.

The regular function in (4.4)

$$
w= \begin{cases}w_{1}=\frac{\partial \chi_{1}}{\partial x}+i \frac{\partial \chi_{1}}{\partial y} & \left(z \in D_{1}\right), \\ w_{2}=\frac{\partial \chi_{2}}{\partial x}+i \frac{\partial \chi_{2}}{\partial y} & \left(z \in D_{2}\right),\end{cases}
$$


is represented by the Cauchy integral as

$$
w_{i}(z)=\frac{1}{2 \pi i} \int_{C_{i}} \frac{w_{i}(Z)}{Z-z} d Z, \quad\left(z \in D_{i}\right),
$$

where $C_{i}$ denotes a closed curve that encircles the region $D_{i}$ (refer to figure 2). It should be noted that $z \notin \partial \Omega\left(\partial \Omega^{ \pm}\right)$in the Cauchy integral (4.8). Taking into account the boundary condition (4.3) at infinity: $w(z) \rightarrow 0(z \rightarrow \infty)$ and imposing the periodicity with respect to $x$, the Cauchy integral (4.8) for $w_{i}=\partial \chi_{i} / \partial x+i \partial \chi_{i} / \partial y$ is reduced to the following line integral:

$$
\begin{aligned}
& \frac{\partial \chi_{i}}{\partial x}(x, y) \\
= & \mp \frac{1}{4 \pi} \int_{-\pi}^{\pi} \frac{\sinh (y-Y)\left(\chi_{i, i n t, x} X_{\theta}-\chi_{i, i n t, y} Y_{\theta}\right)-\sin (x-X)\left(\chi_{i, i n t, x} Y_{\theta}+\chi_{i, i n t, y} X_{\theta}\right) d \theta}{\cosh (y-Y)-\cos (x-X)} \\
& \frac{\partial \chi_{i}}{\partial y}(x, y) \\
= & \mp \frac{1}{4 \pi} \int_{-\pi}^{\pi} \frac{\sin (x-X)\left(\chi_{i, i n t, x} X_{\theta}-\chi_{i, i n t, y} Y_{\theta}\right)-\sinh (y-Y)\left(\chi_{i, i n t, x} Y_{\theta}+\chi_{i, i n t, y} X_{\theta}\right) d \theta}{\cosh (y-Y)-\cos (x-X)},
\end{aligned}
$$

where the upper (lower) sign on the right-hand side of this equation corresponds to $i=2(i=1)$ and we omit the Lagrangian variable $\theta$ in $Z=Z(\theta)=X(\theta)+i Y(\theta)$. Here, the subscript $\theta$ denotes the differentiation with respect to $\theta$ and we used the notation $\chi_{i, i n t, x}=\partial \chi_{i, i n t} / \partial x\left(\chi_{i, i n t, y}=\right.$ $\left.\partial \chi_{i, i n t} / \partial y\right)$

Substituting equations (4.5) and (4.9) into (4.2), we obtain the bulk magnetic field. The magnetic field $\boldsymbol{B}$ in the region $D_{1}\left(D_{2}\right)$ receives the information of that in $D_{2}\left(D_{1}\right)$ through the boundary values $B_{1}^{t}$ and $\nabla \chi_{1, i n t}\left(B_{2}^{t}\right.$ and $\left.\nabla \chi_{2, i n t}\right)$, and evolves under the constraint (2.21). The bulk velocity field $\boldsymbol{u}$ is also provided by the physical quantities on the interface. For the velocity field, refer to Appendix B.

\section{Ideal MHD simulations}

For the purpose of comparison, ideal MHD simulations related to the current-vortex sheet model are performed. Here, we examine two different models which are a shock-induced RMI model and a potential flow model. The initial setup and typical numerical results for the both models are described in this section.

\subsection{Basic equations and numerical scheme}

The basic equations solved in our numerical simulations are the ideal MHD equations. The equation of motion (2.1) and the induction equation (2.2) are used. The compressible fluid is assumed, so that the equation of continuity is

$$
\frac{\partial \rho}{\partial t}+\nabla \cdot(\rho \boldsymbol{u})=0
$$

and the energy equation is

$$
\frac{\partial e}{\partial t}+\nabla \cdot\left[\left(e+p+\frac{B^{2}}{2}\right) \boldsymbol{u}-(\boldsymbol{B} \cdot \boldsymbol{u}) \boldsymbol{B}\right]=0
$$




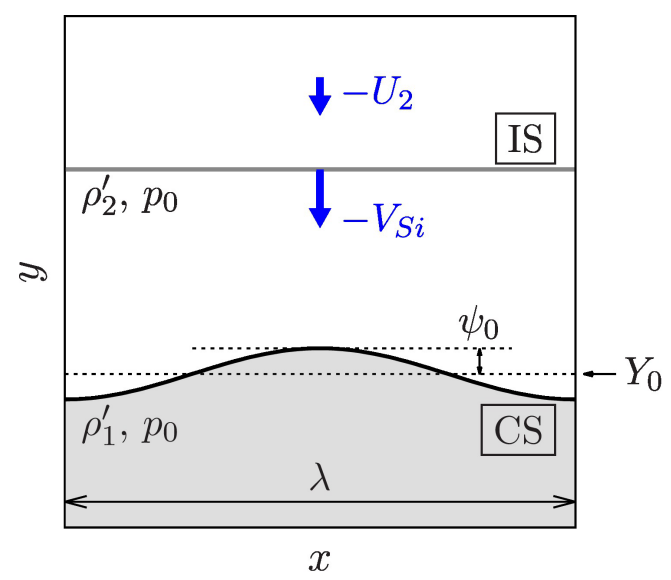

Fig. 3: Schematic picture of the initial configuration for RMI model. Two fluids are divided by a contact discontinuity (CS). An incident shock (IS) propagates in the fluid "2" with the shock velocity $V_{\mathrm{Si}}$.

where $e$ is the total energy density,

$$
e=\frac{p}{\gamma-1}+\frac{\rho u^{2}}{2}+\frac{B^{2}}{2} .
$$

In our simulations, the equation of state for ideal gas is adopted with the adiabatic index $\gamma=5 / 3$.

We solve these ideal MHD equations by using a conservative Godunov-type scheme (e.g., Sano et al., 1998). Operator splitting algorithm is adopted in our scheme. The hydrodynamical part of the equations is solved by a second-order Godunov method, using the exact solutions of a simplified MHD Riemann problem, which includes only the tangential component of a magnetic field. Since the characteristic velocity is then the magnetosonic wave alone, the simplified MHD Riemann problem can be solved in a way similar to the hydrodynamical one (Colella and Woodward, 1984). The piecewise linear distributions of flow quantities are calculated with a monotonicity constraint following van Leer's method (van Leer, 1979). The induction equation is solved by the consistent MoC-CT method (Stone and Norman, 1992, Clarke, 1996), guaranteeing $\nabla \cdot \boldsymbol{B}=0$ within roundoff error throughout the calculation (Evans and Hawley, 1988). All of the MHD simulations in this paper use a grid resolution of $\Delta x=\Delta y=\lambda / 512$, where $\lambda$ is the wavelength of the initial perturbation.

\subsection{RMI model}

First, we explain the RMI model. The initial configuration for our single-mode analysis is illustrated in figure 3. Two fluids with different densities, $\rho_{1}^{\prime}$ and $\rho_{2}^{\prime}\left(<\rho_{1}^{\prime}\right)$, are separated by a contact discontinuity. Here the $x$ - and $y$-axis are set to be parallel and perpendicular to the shock surface. The interface has an initial corrugation of a sinusoidal form, $Y_{\mathrm{cd}}(x)=Y_{0}+\psi_{0} \cos k x$, where $\psi_{0}$ is a corrugation amplitude, $k=2 \pi / \lambda$ is the perturbation wavenumber, and $\lambda$ is the wavelength. This spatial corrugation of the interface is an essential ingredient for the occurrence of the RMI.

A shock propagating through the light fluid "2" $\left(y>Y_{\mathrm{cd}}\right)$ hits the interface at a time $t=0$. The incident shock velocity is $-V_{\mathrm{Si}}$, and the fluid velocity behind the shock is $-U_{2}$. Let $p_{0}$ be a 
uniform pressure of the both fluids before shock passage. The Mach number of the incident shock is defined as $M=V_{\mathrm{Si}} / c_{s 2}^{\prime}$ where $c_{s 2}^{\prime}=\left(\gamma p_{0} / \rho_{2}^{\prime}\right)^{1 / 2}$ is the sound speed of the fluid "2".

The initial magnetic field is uniform with the size of $|\boldsymbol{B}|=B_{0}^{\prime}$ in the preshocked region. As for the field direction, it is assumed to be parallel to the contact surface, so that the field is mostly $B_{x}=B_{0}^{\prime}$ and $B_{y}=0$. However, to avoid the presence of the normal field at the interface, the initial field is calculated from the following vector potential,

$$
A_{z}(x, y)=B_{0}^{\prime} y-B_{0}^{\prime} \psi_{0} \cos k x \exp \left[-k\left|y-Y_{\mathrm{cd}}(x)\right|\right],
$$

by the relation $\boldsymbol{B}=\boldsymbol{\nabla} \times \boldsymbol{A}$.

The initial configuration depicted by figure 3 can be characterized by only four non-dimensional parameters. The sonic Mach number $M$ parameterizes the incident shock velocity. The contact discontinuity is expressed by the density jump $\rho_{1}^{\prime} / \rho_{2}^{\prime}$ and the ratio of the corrugation amplitude to the wavelength $\psi_{0} / \lambda$. The initial field strength is given by the plasma beta $\beta_{0}=2 p_{0} / B_{0}^{\prime 2}$ which is the ratio between the plasma and magnetic pressures defined at the preshocked region.

The linear growth velocity of the RMI can be estimated from the densities and tangential velocities at the interface after the passage of the incident shock (see Appendix E). The densities in the postshocked regions behind the transmitted and reflected shock are $\rho_{1}$ and $\rho_{2}$, respectively, which are calculated from the Rankine-Hugoniot conditions for MHD shocks in the limit of $\psi_{0} / \lambda \ll$ 1. The transmitted and reflected shock velocities, $V_{S T}$ and $V_{S R}$, and the contact surface velocity $U$ can also be evaluated from the same MHD Riemann problem. With the help of equations (E1) and (E2), the tangential velocity at the interface can be obtained from the initial parameters of the RMI model. The growth velocity is then given by equation (E5).

The time evolution of a fiducial RMI model is depicted by figure 4, which shows the spatial distribution of normalized density $\rho / \rho_{2}$, current density $(\boldsymbol{\nabla} \times \boldsymbol{B})_{z} /\left(k \bar{B}_{0}\right)$, and vorticity $(\boldsymbol{\nabla} \times$ $\boldsymbol{u})_{z} /\left(k v_{\text {lin }}\right)$ at $k v_{\text {lin }} t=4$. The average field $\bar{B}_{0} \equiv\left(B_{1}+B_{2}\right) / 2$ and the linear growth velocity are used for the normalization, where $B_{1}$ and $B_{2}$ are the postshock field strength at the interface.

To compare the results with those of the sheet model, it is useful to label the RMI model by the Atwood number $A$ and the Alfvén (Mach) number $R_{A}$ defined by

$$
R_{A} \equiv \sqrt{\frac{\rho_{1}+\rho_{2}}{2}} \frac{v_{l i n}}{\bar{B}_{0}}=\frac{v_{l i n}}{v_{a 0}},
$$

where $v_{a 0}$ denotes the Alfvén velocity (3.5). These parameters for the model shown by figure 4 are $A=-0.2$ and $R_{A}^{2}=10^{3}$. Notice that $A$ and $R_{A}$ are estimated from the postshock quantities, which are different from the initial parameter for the RMI model. For this particular model, the initial density ratio is $\rho_{1}^{\prime} / \rho_{2}^{\prime}=1.55$, the Mach number of the incident shock is $M=2$, the corrugation amplitude is $\psi_{0} / \lambda=0.05$, and the initial plasma beta is $\beta_{0}=8.85 \times 10^{-7}$.

Mushroom-shaped spike growth can be seen in the density distribution of figure 4(a). The interface is largely deformed by the RMI growth. The distance from the spike top to bubble bottom is about $0.6 \lambda$ that is much larger than the initial amplitude $\psi_{0}=0.05 \lambda$. Magnetic field lines drawn over the density profile are obviously stretched by the RMI motions, but the field is always parallel to the interface. In ideal MHD, the current density is given by $\boldsymbol{\nabla} \times \boldsymbol{B}$. As seen from figure $4(\mathrm{~b})$, the current density is localized near the interface and the bulk component can be negligible for this model. The vorticity is also concentrated at the interface and the peak is located at the core of the mushroom shape. In fact, the bulk vorticity can be generated behind 


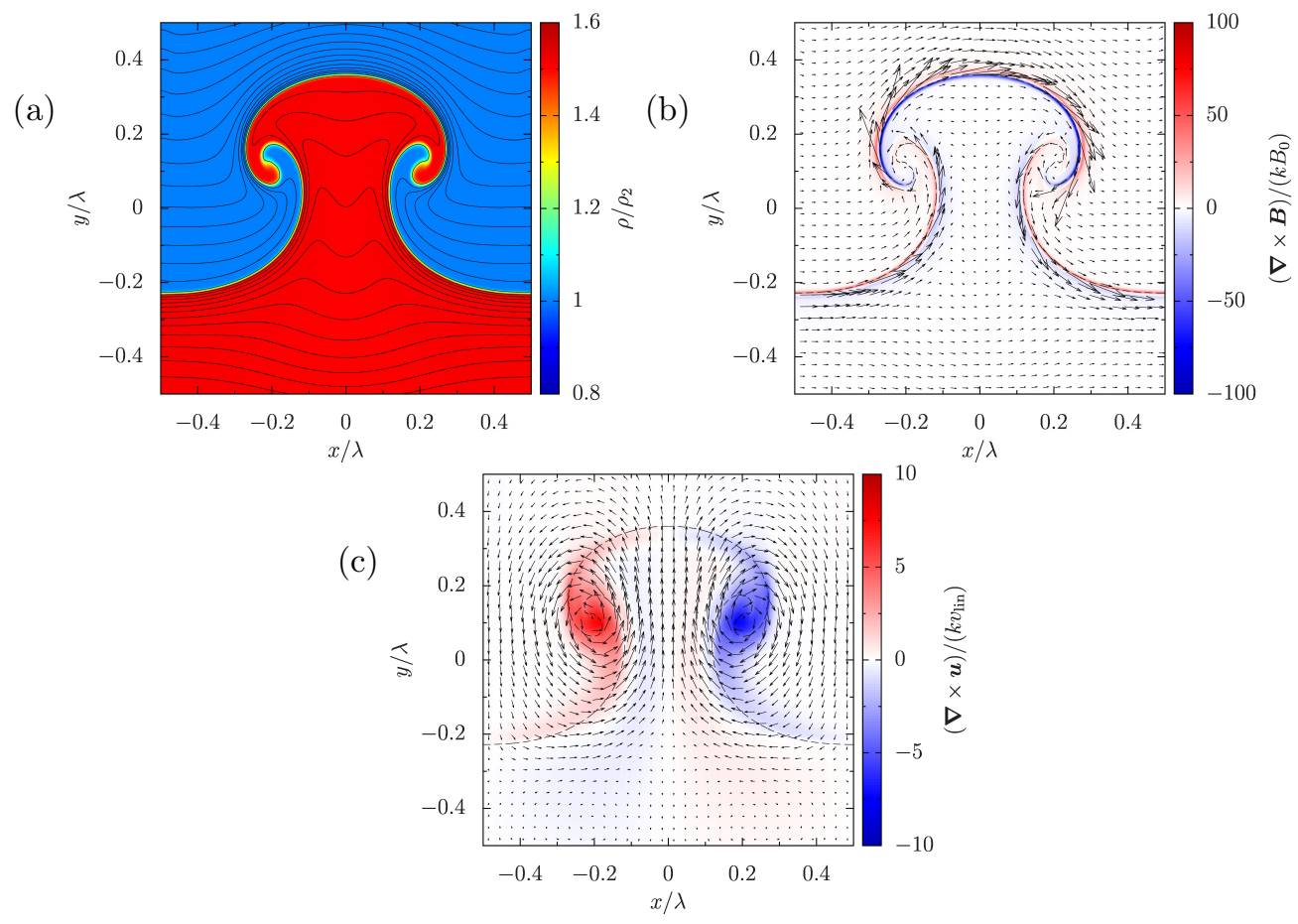

Fig. 4: Normalized plasma density with magnetic field lines (upper left), current density (upper right), and vorticity (lower) at $k v_{l i n} t=4.0$ obtained by the ideal MHD simulation of a RMI model, where $A=-0.2$ and $R_{A}^{2}=10^{3}$. Magnetic field lines are shown over the density contour. The arrows in the current density and vorticity figures are the magnetic field and velocity vectors, respectively. 
the rippled transmitted and reflected shocks. However, for the weak shock cases $M \lesssim 2$, the bulk vorticity can be ignored compared to the dominant value at the interface. For the strong shock cases, on the other hand, it is known that the bulk vorticity can reduce significantly the growth velocity of the RMI (Wouchuk, 2001). Thus the sheet model can be applicable only for the RMI models in the weak shock limit.

\subsection{Potential flow model}

The second model to be performed is a potential flow model, which is a simplified setup to mimic the behavior of RMI. The advantage of this model is that it is possible to start from almost the same initial conditions as the current-vortex sheet model. In the potential flow model, a flat vortex sheet is assumed initially at $y=0$. The velocities at $y>0$ are set to be

$$
\begin{aligned}
& u_{x}=v_{l i n} \sin k x \exp (-k y), \\
& u_{y}=v_{l i n} \cos k x \exp (-k y),
\end{aligned}
$$

and, if $y<0$ then

$$
\begin{gathered}
u_{x}=-v_{l i n} \sin k x \exp k y, \\
u_{y}=v_{l i n} \cos k x \exp k y .
\end{gathered}
$$

Thus the velocity jump at the interface is initially given by

$$
\Delta u_{x}=-2 v_{l i n} \sin k x
$$

Note that in this model $v_{l i n}$ is just a parameter. The density is set to be uniform with the size of $\rho_{2}\left(\rho_{1}\right)$ for $y>0(y<0)$. In terms of the field geometry, a uniform field parallel to the interface is assumed, that is

$$
B_{x}=B_{0} \text { and } B_{y}=0 .
$$

Figure 5 shows the result of ideal MHD simulations for a potential flow model. The Atwood number and Alfvén number in a potential flow model can be easily calculated from the initial densities $\rho_{1}$ and $\rho_{2}$, shear size $v_{\text {lin }}$, and field strength $B_{0}$. A model shown in figure 5 is for the case of $A=-0.2$ and $R_{A}^{2}=10^{3}$, which are the identical to those in figure 4 for the RMI model. The spacial distribution of the density, velocity, and magnetic field in the RMI model (figure 4) and the potential flow model (figure 5) are quite similar. The location of the spike top and bubble bottom are almost the same, so that the growth velocity of the RMI can be reproduced accurately by the potential flow model. The localized features in the current density and vorticity are also captured by the simple potential flow model. An obvious difference is in the tightness of the roll-up of the mushroom shape, which might be affected by the numerical diffusion during the interaction of the incident shock and interface in the RMI model, and also bulk vorticity behind the transmitted shock that decays with time.

The unstable motions of the RMI stretches the interface as well as the magnetic field lines, which brings the amplification of the magnetic fields during the RMI growth. Figure 6 shows the time evolution of the maximum field strength. We examined the dependence on the density jump at the interface, e.g., the Atwood number $A$, and the initial field strength, or the Alfvén number $R_{A}$. If the initial field is weaker, the magnetic field increases efficiently and saturates in a few 
(a)

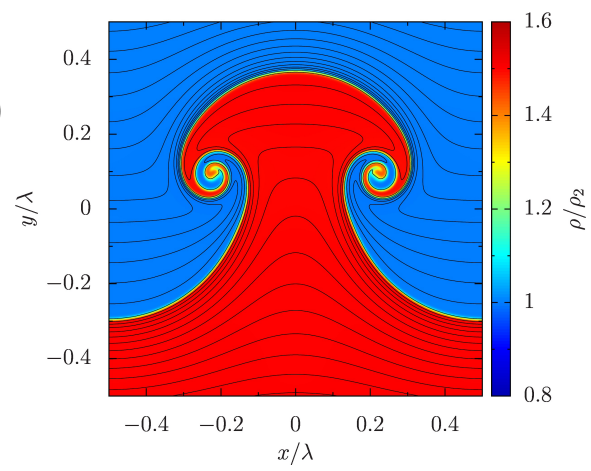

(b)

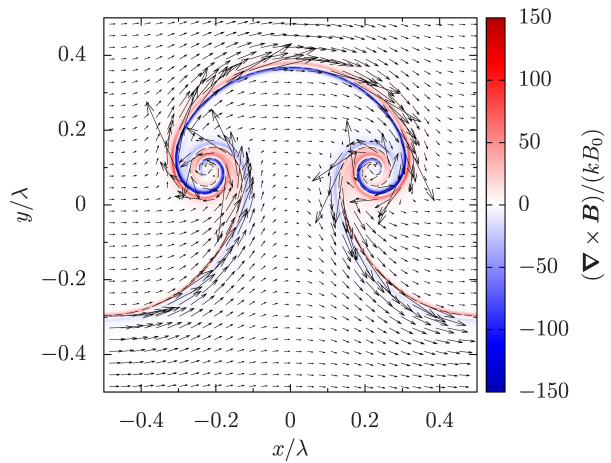

(c)

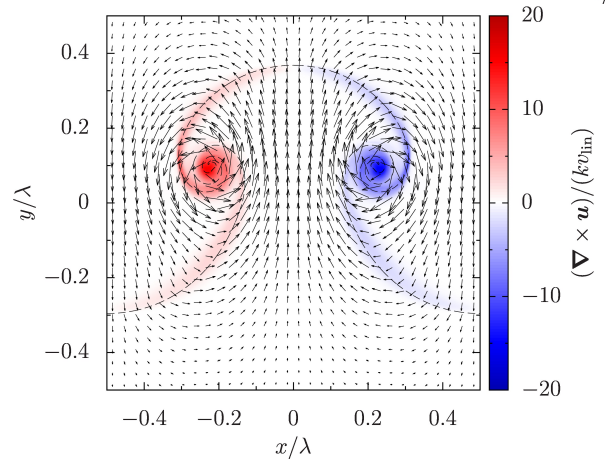

Fig. 5: Normalized plasma density with magnetic field lines (upper left), current density (upper right), and vorticity (lower) at $k v_{l i n} t=4.0$ obtained by the ideal MHD simulation of a potential flow model, where $A=-0.2$ and $R_{A}^{2}=10^{3}$. The meaning of the lines and arrows in the figures are the same as in figure 4.

(a)

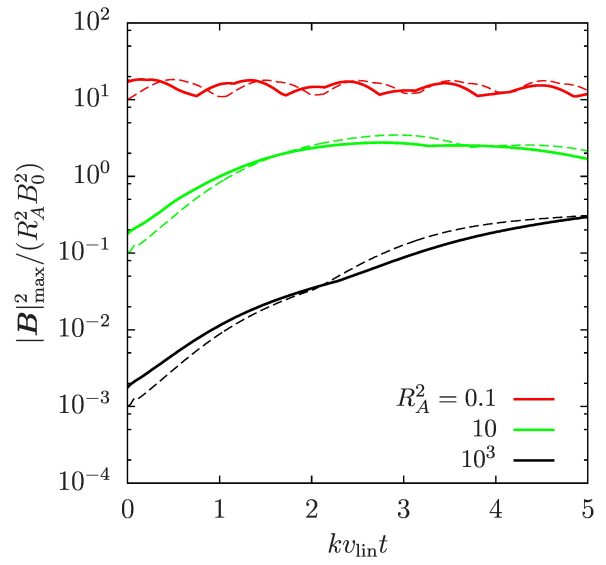

(b)

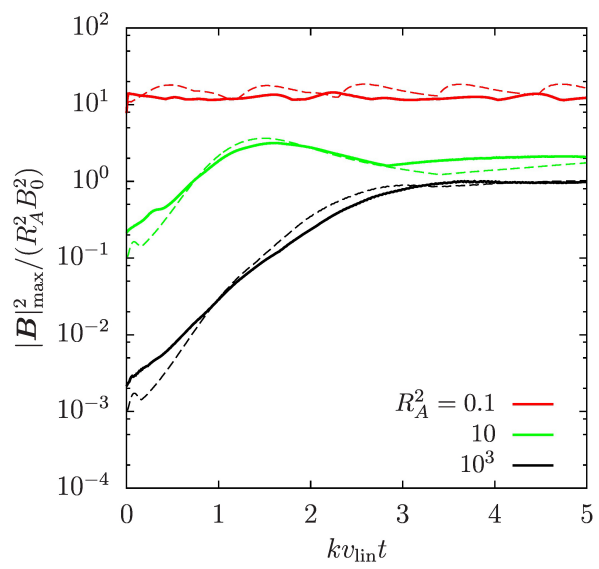

Fig. 6: Growth of the maximum value of $|\boldsymbol{B}|^{2} /\left(R_{A}^{2} B_{0}\right)^{2}$ by the ideal MHD simulations for $A=$ (a) -0.2 and (b) -0.7 , where the dotted and solid lines denote the potential flow and shock induced RMI, respectively. 
(a)

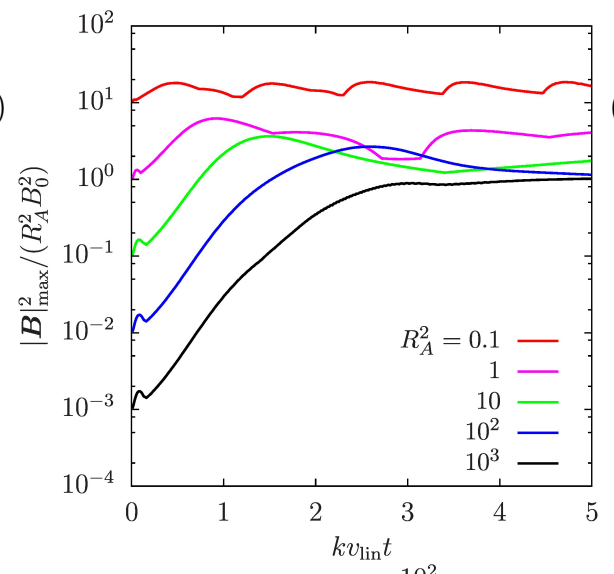

(b)

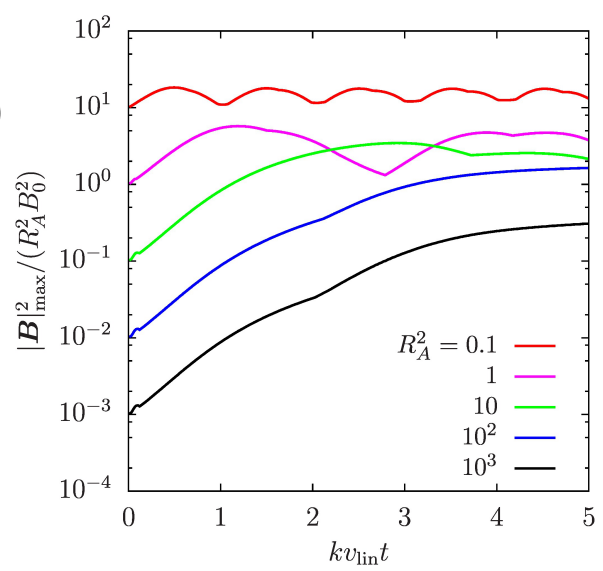

(c)



Fig. 7: Growth of the magnetic field by the ideal MHD potential flow simulation. The vertical axis denotes the maximum value of $|\boldsymbol{B}|^{2} /\left(R_{A}^{2} B_{0}\right)^{2}$, where the Atwood number $A=(\mathrm{a})-0.7$, (b) -0.2 , and (c) 0 . 
times of $k v_{l i n} t$. When the magnetic field is strong enough, the maximum value oscillates around the initial strength.

In this figure, the solid curves are the results of the potential flow model and dashed curves are those of the RMI model. Both of the simulations provide almost the same result for any Alfvén numbers and Atwood numbers, which strongly suggests that the potential flow model contains the characteristic features of the RMI surprisingly well. Because the initial setup of the potential flow model is quite similar to that of the sheet model, the comparison with the sheet model results would be quite interesting to demonstrate the validity and accuracy of the sheet model. For this purpose, we calculated the field amplification behavior with various parameters for the potential flow models. Figure 7 shows the models with $A=-0.2,-0.7$ and 0 . The models of $A=0$ has no density jump at the vortex sheet, so that there is no counterpart of the RMI model. The detail comparison with the sheet models is discussed in the next section.

\section{Numerical calculations by the sheet model}

\subsection{Initial conditions and numerical methods for the sheet model}

Most of the numerical calculations of the sheet model are performed for the initial conditions of a sinusoidal vortex sheet strength and a flat interface with no surface current density $\boldsymbol{j}_{s}=0$

$$
\begin{aligned}
& \gamma=-2 v_{l i n} \sin k x \\
& k x=\theta, \quad k y=0, \quad(-\pi \leq \theta \leq \pi) \\
& \boldsymbol{B}_{i} \equiv \boldsymbol{B}_{0}=B_{0} \hat{\boldsymbol{e}_{x}}(i=1,2) .
\end{aligned}
$$

It should be noted that $v_{l i n}$ corresponds to the initial velocity shear given by equation (E.5) (for the derivation of $v_{l i n}$, refer to Appendix E) for RMI in the weak shock limit, in which $\delta v_{1,2}^{*}$ represent the transverse velocities immediately after the shock-interface interaction. In the subsequent calculations, we use the following normalizations: $k \boldsymbol{x} \rightarrow \boldsymbol{x}, k v_{l i n} t \rightarrow t, k \Gamma / v_{l i n} \rightarrow \Gamma, \gamma / v_{l i n} \rightarrow \gamma$, and $\boldsymbol{B}_{i} / B_{0} \rightarrow \boldsymbol{B}_{i}$. Using the Alfvén number, the last term of (2.29) is rewritten as

$$
\frac{2}{s_{\theta}\left(\rho_{1}+\rho_{2}\right)}\left(\langle\boldsymbol{B}\rangle \cdot \boldsymbol{j}_{s}\right)_{\theta} \quad \rightarrow \quad \frac{1}{s_{\theta} R_{A}^{2}}\left(\langle\boldsymbol{B}\rangle \cdot \boldsymbol{j}_{s}\right)_{\theta}
$$

and the square of it describes the ratio of the magnitude of the magnetic force to the inertia. It should be mentioned that two parameters, the Atwood number $A$ and the Alfvén number $R_{A}$, determine the nonlinear dynamics of a current-vortex sheet for this initial value problem.

We adopt the vortex blob method for the computations of the model equations. This method is developed by Krasny, in which he introduced a regularized parameter $0<\delta \ll 1$ in the denominator of the Birkhoff-Rott equation (2.13) to avoid the divergence of the integral (Krasny, 1987). The parameter $\delta$ plays an important role in cutting higher-order Fourier modes, which enables us to perform the long-time calculation of vortex sheet motion such as roll-ups. For the periodic case, the Birkhoff-Rott equation (2.13) by this regularization is given as

$$
\begin{aligned}
U & =-\frac{1}{4 \pi} \int_{-\pi}^{\pi} \frac{\sinh \left(Y(\theta, t)-Y\left(\theta^{\prime}, t\right)\right) \gamma\left(\theta^{\prime}, t\right)}{\cosh \left(Y(\theta, t)-Y\left(\theta^{\prime}, t\right)\right)-\cos \left(X(\theta, t)-X\left(\theta^{\prime}, t\right)\right)+\delta^{2}} d \theta^{\prime} \\
V & =\frac{1}{4 \pi} \int_{0}^{2 \pi} \frac{\sin \left(Y(\theta, t)-Y\left(\theta^{\prime}, t\right)\right) \gamma\left(\theta^{\prime}, t\right)}{\cosh \left(Y(\theta, t)-Y\left(\theta^{\prime}, t\right)\right)-\cos \left(X(\theta, t)-X\left(\theta^{\prime}, t\right)\right)+\delta^{2}} d \theta^{\prime}
\end{aligned}
$$


When the regularized parameter $\delta=0$, the parameter $\tilde{\alpha}$ should be taken to $\tilde{\alpha}=A$; however, there is arbitrariness in selecting $\tilde{\alpha}$ when $\delta \neq 0$ (Matsuoka and Nishihara, 2006). This arbitrariness comes from arbitrariness in the tangential velocity of the interface (Pullin, 1982, Kerr, 1988). The introduction of $\delta$ gives an artificial thickness to the interface. We select as $\delta=0.15$ throughout the calculations by the sheet model. The spatial integration of (6.2) can be carried out using the conventional trapezoidal rule. For the temporal integration, we use the fourth-order Runge-Kutta scheme. The Fredholm equation of the second kind (2.29) is solved by iteration until convergence within some tolerance level (here, we select the level as $10^{-9}$ ). In order to cut the irregular motion due to the round-off error that increases with the number of grid points $N$, we use the filtering technique introduced by Krasny (Krasny, 1986). The filter level depends on the spatial resolution, i.e., the number of grid points $N$ and in general, the higher filter levels are demanded for the larger $N$. Here, we select as $N=512$ and the filter level as $10^{-13}$. We use the grid redistribution method by the Newtonian method (Baker and Nachbin, 1998, Matsuoka and Nishihara, 2006) for long-time computations; however, all results including the breakdown time of the computation are unchanged even if we adopt the point insertion scheme (Krasny, 1987, Matsuoka, 2013). Details of the numerical methods to solve equations (2.12), (2.29), and (6.2) are presented in Appendix C. In the subsequent subsections, we compare the non-uniform current-vortex sheet model with ideal MHD simulations of a potential flow.

Remark 1. The theory for the magnetic field in the bulk presented in section 4 is mathematically constructed under the condition that the regularized parameter $\delta=0$. This theory is applicable to the bulk magnetic field for $\delta \neq 0$ as well by adopting the evaluation method for the multivalued interface presented in Appendix D. However, it should be noted that the bulk magnetic field provided by this method is an approximation and it is not the exact solution because the sheet model for $\delta \neq 0$ is not the exact solution of the 2D Euler system (Holm et al., 2006).

Remark 2. When $\delta=0$, the method of conformal mapping is also applicable for describing the bulk magnetic field; however, this method cannot use for the case of $\delta \neq 0$; i.e., for the case of multi-valued interfaces as presented in the current paper.

\subsection{Comparison of sheet model calculations with ideal MHD simulations}

We first compare the results obtained from the sheet model with those from the ideal MHD simulations. The initial conditions of the model calculation are as follows; consider a flat interface with a sinusoidal vortex sheet strength given by equation (6.1) for the parameters of the Atwood number $A=-0.2$ and the Alfvén number of $R_{A}^{2}=10^{3}$. These initial conditions correspond to the case discussed in subsection 5.3, namely for the initial potential flow. The left figure in figure 8 shows the interface shape with the current sheet strength $j_{s}$ and the magnetic field in the bulk indicated by arrows at time of $k v_{l i n} t=4.0$, while the arrows in the right figure present the velocity in the bulk. We used the time step $\triangle t=0.0005$ and $\tilde{\alpha}=A^{2}$ for the calculations of $A=-0.2$, while $\triangle t=0.00025$ and $\tilde{\alpha}=0.05$ for $A=-0.7$, and the number of grid points $N$ was set to $N=512$. The bulk magnetic field and velocity field are calculated from the variables on the interface as discussed in section 4 and Appendix B.

The corresponding results obtained by the ideal MHD simulations are shown in figure 5 . As expected, both results (sheet model and MHD simulation) show the growth of the spike in the $y$-direction due to RMI as indicated by the velocity arrows. In the nonlinear stage, a mushroom 

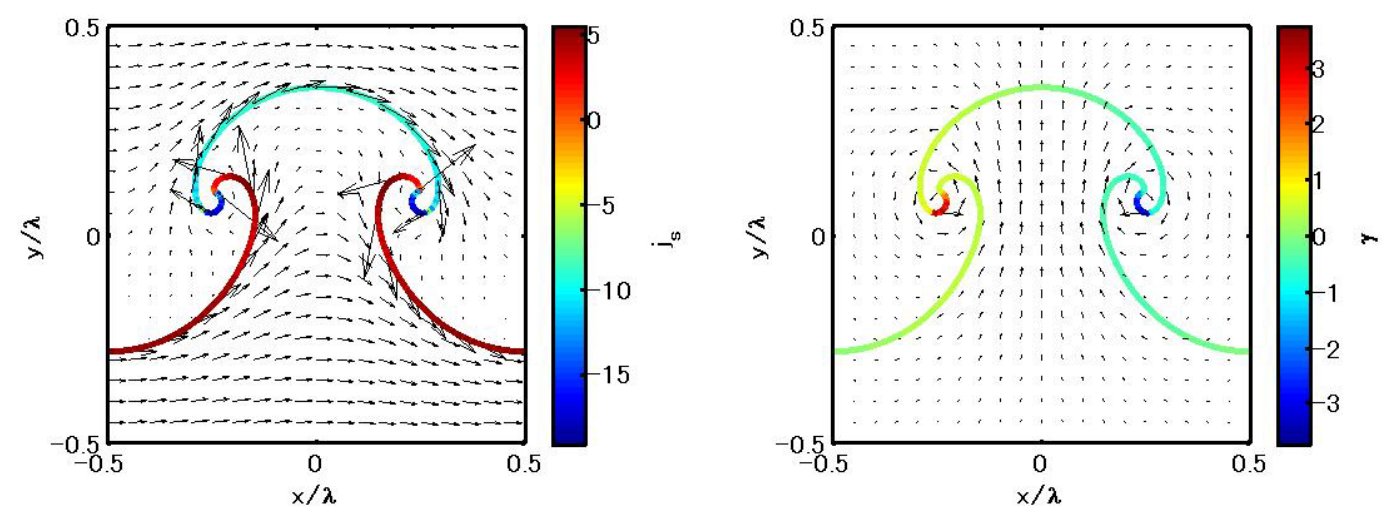

Fig. 8: Bulk magnetic field (arrows) in the left and velocity field (arrows) in the right at $k v_{l i n} t=4.0$ obtained by the sheet model, where $A=-0.2$ and $R_{A}^{2}=10^{3}$. The color bar in the left (right) figure denotes the current sheet strength $j_{s}$ (vortex sheet strength $\gamma$ ).

shape of the spike appears and also the roll-up structure is observed in the tip of the mushroom in both results. The roll-up for the corresponding Atwood number is weaker than that of a pure vortex sheet (Matsuoka and Nishihara, 2006) due to the magnetic tension.

The model shows the generation of the non-uniform surface current on the interface as shown by the colored scale in figure 8 . It should be noted that the current sheet strength $j_{s}=0$ initially and the non-uniform surface current is induced accompanied with the interface deformation. We would like to mention that we use the current sheet strength $j_{s}$ and the vortex sheet strength $\gamma$ on the interface as variables in the current-vortex sheet model. On the other hand, the magnetic field and velocity field on the grid points (mostly in the bulk) are used instead of these quantities in the ideal MHD simulations because $j_{s}$ and $\gamma$ cannot define. In the MHD simulation we can estimate the current density from the relation of $\boldsymbol{j}(x, y)=\nabla \times \boldsymbol{B}(x, y)$, which is shown in figure 5 with the colored scale. Note that the value of $\boldsymbol{j}$ strongly depend on the mesh size used in the estimation. However, the current density thus estimated are localized near the interface, which corresponds to the surface current density $\boldsymbol{j}_{s}$ in the model. By comparing figures 5 and 8 , one can see that the roll-up is not as extensive in the sheet model as in the potential flow model. The strength of roll-ups can be controlled by $\delta$. When we reduce the regularized parameter $\delta$, the roll-up becomes tighter (Matsuoka and Nishihara, 2006).

The strong magnetic field is observed near the interface in the MHD simulation as shown in figure 5. That is not visible in the sheet model because of large mesh size used in the calculation of the bulk magnetic field from the interface values. However, we can directly show the magnetic field $\boldsymbol{B}_{1}$ (magnetic field in the high density side) and $\boldsymbol{B}_{2}$ (magnetic field in the low density side) on the interface are shown in figure 9 (upper two figures). The maximum magnetic field is amplified more than 30 times, which is large enough to explain the generation of the high energy cosmic protons in SNRs. The corresponding current sheet strength and vortex sheet strength are also shown for the same parameters and at the same time in figure 9. The maximum values of $|\gamma|$ in figure 9 appears in the neighborhood of vortex cores such as a vortex pair as found in the pure hydrodynamic RMI (Matsuoka and Nishihara, 2006).

The model calculations and ideal MHD simulations agree fairly well with each other as a whole, 

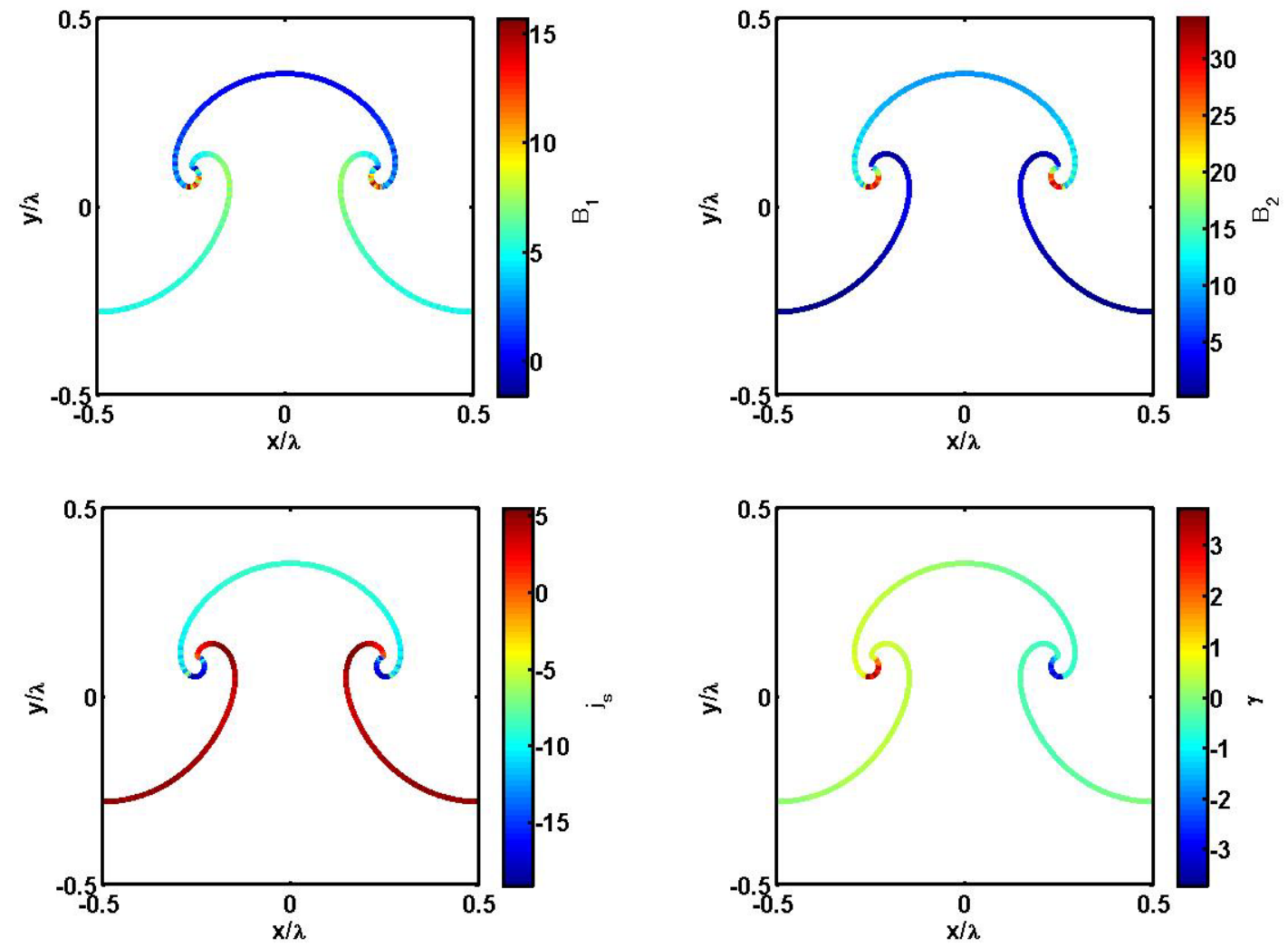

Fig. 9: Magnetic field $B_{1}^{t}$ (upper left), magnetic field $B_{2}^{t}$ (upper right), current sheet strength $j_{s}=B_{1}^{t}-B_{2}^{t}$ (lower left), and vortex sheet strength $\gamma$ (lower right) for $A=-0.2$ at the normalized time $k v_{\text {lin }} t=4.0$, where $R_{A}^{2}=10^{3}$ and the upper fluid $\left(\rho_{2}\right)$ is assumed to be lighter than the lower one $\left(\rho_{1}\right)$. The spatial scale is normalized by $2 \pi$. 

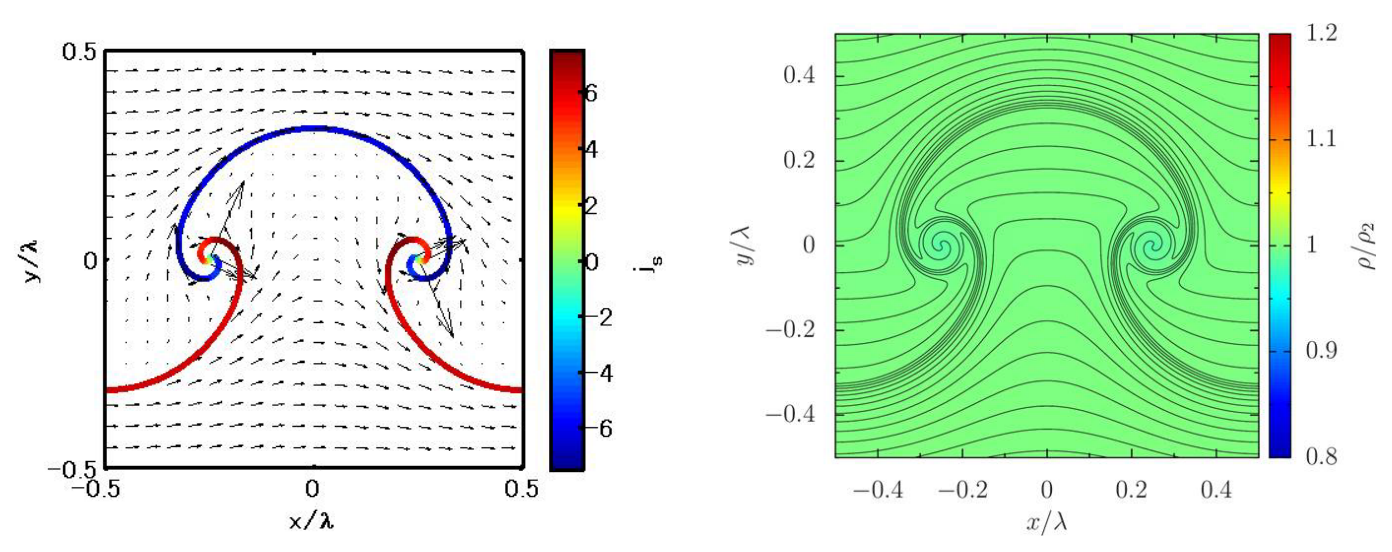

Fig. 10: Bulk magnetic field (arrows) by the sheet model (left) and the magnetic field line by the the ideal MHD simulation of the potential field (right) at $k v_{l i n} t=4.0$, where $A=0$ and $R_{A}^{2}=10^{3}$. The color bars in the left and right figure denote the current sheet strength $j_{s}$ and the scaled density, respectively.

including the formation of mushroom and spiral structures and the magnetic field amplification, even though they use different variables either on the interface (1D variables) or in the bulk (2D variables), respectively. It should also be mentioned that the bulk magnetic field and bulk velocity field are obtained from the interface variables in the current-vortex sheet model, and they also agree with those obtained by the ideal MHD simulations.

\subsection{Atwood number dependence on nonlinear evolution of the sheet}

Before showing the magnetic field amplification, we present briefly the dependence of the interfacial dynamics on the Atwood number, since the interfacial dynamics determines the local magnetic field amplification on the sheet through the induction equation of equation (2.24). Figure 10 shows the spatial profile of the spike and the magnetic field (line) at the same time of $k v_{\text {lin }} t=4.0$ for the Atwood number of $A=0$ obtained from the model (left) and the ideal MHD simulation (right), respectively. There is no density jump across the current-vortex sheet in the case of $A=0$; therefore, the color scale of the mass density is mono-color in the right figure. Moreover, in the case of $A=0$, there is no distinction between bubble and spike and they are symmetrical with respect to the $x$ axis. The shape of the interface for $A=-0.7$ are shown with the tangential magnetic fields at the interface in the high density side $B_{1}^{t}$ and the low density side $B_{2}^{t}$, the current sheet strength $j_{s}$, and the vortex sheet strength $\gamma$ in figure 11. As seen from these figures and compare with figure 9 , the difference of the interface structure can be seen; for example, the strong spiral winding can be observed in small absolute values of the Atwood number and the height of the spike is higher for larger $|A|$. This general feature of the interfacial dynamics depending on the Atwood number agrees with the ideal MHD simulations at least qualitatively. It was also observed very strong spiral winding in pure hydrodynamic experiments for a very small Atwood number (Jacobs and Sheeley, 1996). In the model calculation, the mushroom structure does not appear at this time yet for the Atwood number of $A=-0.7$ as shown in figure 11. Even though no spiral appears, the relatively large current sheet strength $j_{s}$ and thus the relatively large magnetic field 

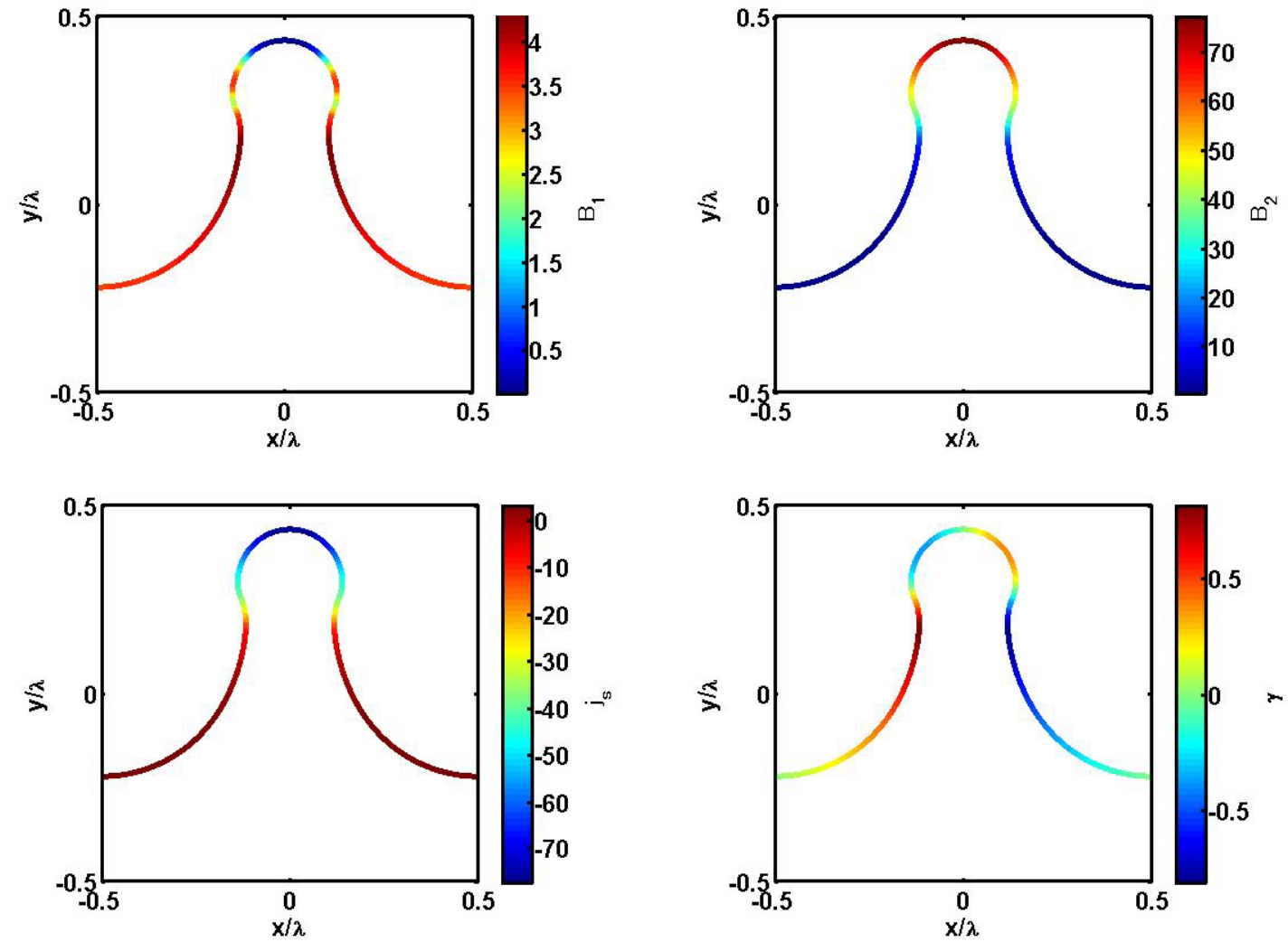

Fig. 11: Magnetic field $B_{1}^{t}$ (upper left), magnetic field $B_{2}^{t}$ (upper right), current sheet strength $j_{s}=B_{1}^{t}-B_{2}^{t}$ (lower left), and vortex sheet strength $\gamma$ (lower right) for $A=-0.7$ at the normalized time $k v_{\text {lin }} t=4.0$, where $R_{A}^{2}=10^{3}$ and the upper fluid $\left(\rho_{2}\right)$ is assumed to be lighter than the lower one $\left(\rho_{1}\right)$. The spatial scale is normalized by $2 \pi$. 

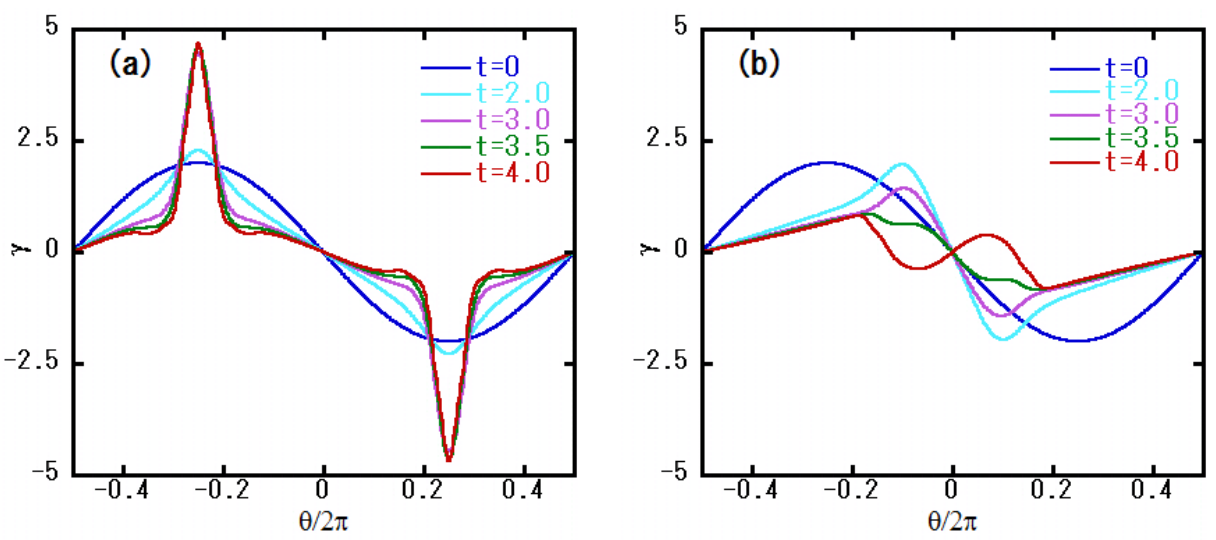

Fig. 12: Vortex sheet strength $\gamma$ versus Lagrange parameter $\theta / 2 \pi(-\pi \leq \theta \leq \pi)$ for $A=($ a) 0 and (b) -0.7, where $R_{A}^{2}=10^{3}$. The phase of $\gamma$ is inverted in the region $3.5 \leq t \leq 4.0$ when $A=-0.7$.

of $B_{2}^{t}$ are observed as shown in the figure.

We depict the temporal evolution of spatial profiles of $\gamma$ for $A=0$ and $A=-0.7$ in figure 12 , taking the case of $R_{A}^{2}=10^{3}$ as an example. We note that the phase of $\gamma$ initially given by $\gamma \propto-\sin \theta$ is inverted between $t=3.5$ and 4.0 in the region of $|\theta| /(2 \pi)<1 /(2 \pi) \sim 0.16$ for $A=-0.7$. In this time duration, the maximum amplitude of $\gamma$ once becomes very small; however, the interface itself is not inverted at this time. The inversion of $\gamma$ is caused by the magnetic tension with opposite phase to $\gamma$, which acts to straighten the interface. On the other hand, the phase inversion of $\gamma$ does not appear for $A=0$ as found in figure 12 (a). When the absolute value of the Atwood number is small and the Alfvén number $R_{A}$ is large, the roll-up of the interface occurs and the vortex sheet strength $\gamma$ at the cores becomes very large. Then the inertial force for rolling up is stronger than the force by the magnetic tension; therefore, the phase inversion of $\gamma$ does not occur for this case.

\subsection{Magnetic field amplification}

As has been shown in section 5, the large amplification of the magnetic field has been observed in ideal MHD simulations for the various Atwood numbers and Alfvén numbers. Figures 13 (a), (b), and (c) show the corresponding magnetic field amplification observed in the model calculations for the different Atwood numbers of (a) $A=-0.7$, (b) $A=-0.2$, and (c) $A=0$ with the various initial Alfvén numbers from $R_{A}^{2}=0.1$ to $10^{3}$. Figure 13 shows the maximum values of $\left|\boldsymbol{B} / \boldsymbol{B}_{0}\right|^{2}$ multiplied by $R_{A}^{-2}$. We see that the magnetic field is always amplified when the value of $R_{A}$ is large $\left(R_{A}^{2} \geq 10\right)$ regardless of the Atwood number. The normalized magnetic field $|\boldsymbol{B}|^{2} /\left(R_{A}^{2} B_{0}\right)^{2}$ grows exponentially in time at the earlier stage for all Atwood numbers. This exponential growth of the magnetic field is due to the stretching of the interface (Sano et al., 2013). The model results show very much similar growth of the magnetic field in time as those obtained by the ideal MHD simulations shown in figures 7 for any values of the two parameters. On the other hand, when the Alfvén number $R_{A}$ is small, the maximum magnetic field intensity oscillate for all Atwood numbers, and its amplification is not observed. This oscillation is found to be due to the surface Alfvén wave as will be discussed in subsection 6.5 later. 

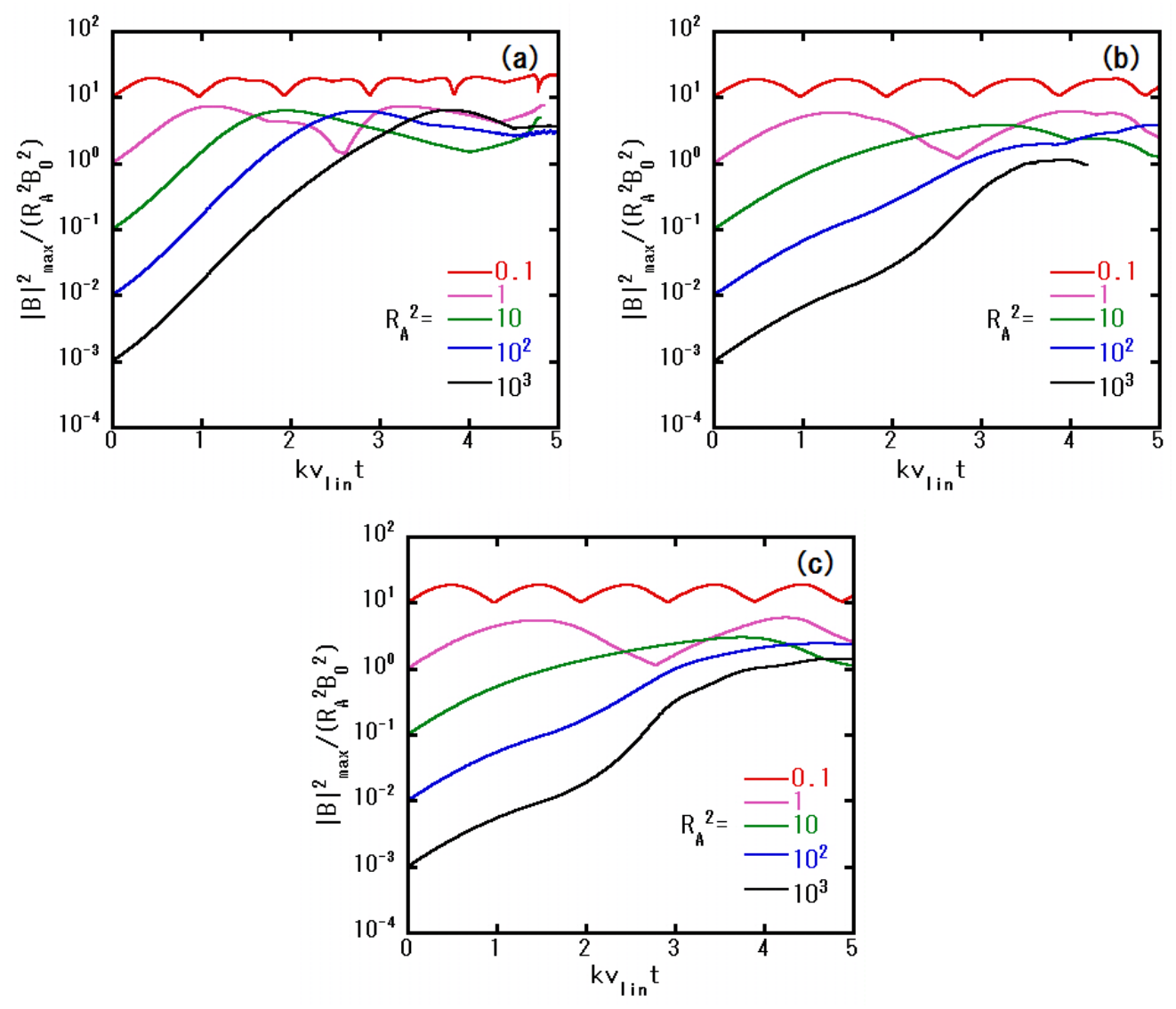

Fig. 13: Growth of the magnetic field by the sheet model. The vertical axis denotes the maximum value of $|\boldsymbol{B}|^{2} /\left(R_{A}^{2} B_{0}\right)^{2}$, where the Atwood number $A=$ (a) -0.7 , (b) -0.2 , and (c) 0 . 



Fig. 14: Interfacial magnetic fields $B_{1}^{t}$ and $B_{2}^{t}$ versus normalized Lagrange parameter $\theta / 2 \pi(-\pi \leq$ $\theta \leq \pi)$ at $k v_{l i n} t=4.0$ for $A=(\mathrm{a}) 0$ and (b) -0.7 , where $R_{A}^{2}=10^{3}$.

When the maximum magnetic field grows exponentially, the larger of the absolute values of Atwood number $|A|$ result in the larger growth rate. This can be due to the larger nonlinear growth of the spike for the larger Atwood number; i.e., this can be seen by comparing the spike height for the different Atwood numbers as discussed in the previous subsection 6.3, even though the initial vortex sheet strength are the same for every case. This can be understood as follows; even if we gave the same initial velocity shear (vortex sheet strength); i.e., the same linear growth rate of RMI, the spike can be penetrate into the light fluid more easily for the cases of the large Atwood number in the nonlinear stage, since the ratio of the heavy fluid mass density (region 1) to the light fluid mass density (region 2) is larger for the larger absolute value of the Atwood number.

When the absolute value of the Atwood number is small $(|A|=0,0.2)$, a kind of inflection point appears in the growth of the maximum magnetic field intensity for large Alfvén numbers $\left(R_{A}^{2} \geq 10^{2}\right)$ at around $k v_{l i n} t=2.0-3.0$ as shown in figure 13 (b) $A=-0.2$ and (c) $A=0$. However, this does not occur for the case of (a) $A=-0.7$. It is found that at this inflection point of the growth, the spatial positions where the magnetic field on the interface is maximized shifts from the top of the spike to the neighborhood of the core of the spiral, more exactly the outer sides of the mushroom. Figure 14 (a) and (b) show the interfacial value of the magnetic fields $B_{1}^{t}$ and $B_{2}^{t}$ versus Lagrange parameter $\theta / 2 \pi(-\pi \leq \theta \leq \pi)$ at $k v_{\text {lin }} t=4.0$ for large $(|A|=0.7)$ and small $(A=0)$ Atwood numbers, where $R_{A}^{2}=10^{3}$. When $A=0$, the magnetic fields $B_{1}^{t}$ and $B_{2}^{t}$ are identical if we exchange the bubble and spike $(\theta /(2 \pi)= \pm 0.5$ and $\theta /(2 \pi)=0)$, while the value of the magnetic field $B_{2}^{t}$ at the spike $(\theta /(2 \pi)=0)$ is much larger than the one of $B_{1}^{t}$ in figure 14 (b). The maximum value of the magnetic field at this time appears at the vortex cores $(\theta \sim \pm \pi / 4)$ for $A=0$ and that appears at the spike in the magnetic field of the lighter fluid $B_{2}^{t}$ for $A=-0.7$. These points are the places that the interface is most stretched and the magnetic field is magnified there.

The stretching rate of the interface $G_{s}(t)$ defined by

$$
G_{s}(t)=\int \frac{\partial\left(\boldsymbol{u}_{2} \cdot \boldsymbol{t}\right)}{\partial s} \mathrm{~d} t
$$

is plotted as a function of the Lagrange parameters $\theta /(2 \pi)$ at time $k v_{\text {lin }} t=$ (a) 1.5 and (b) 4.0 for the Atwood number of $A=0$ and $A=-0.7$ in figure 15. As clearly seen in figure 15 (a), at the 

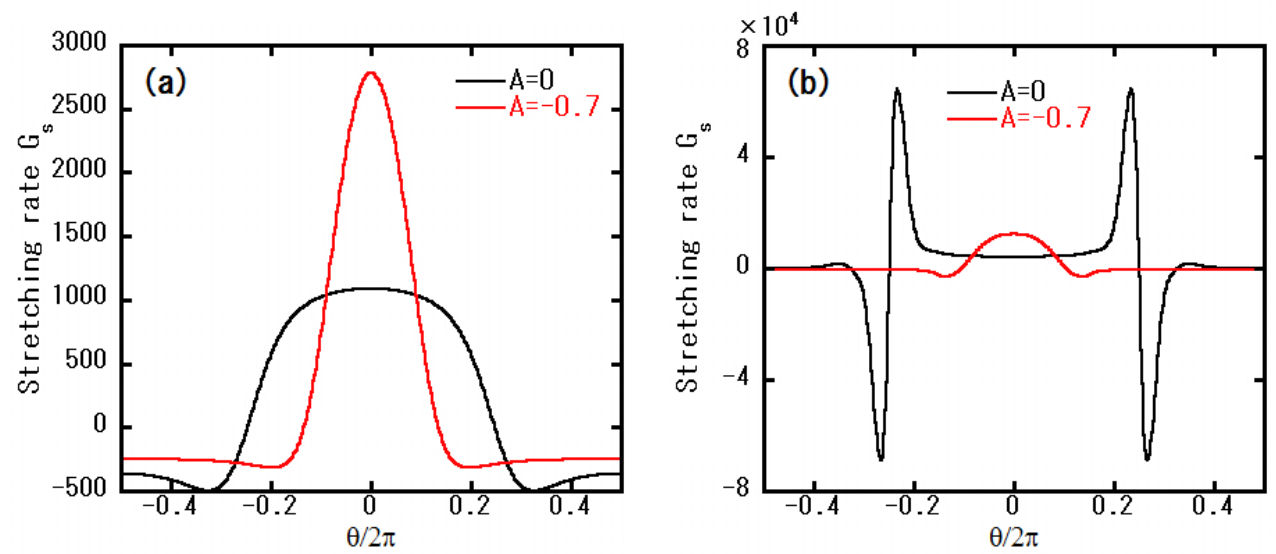

Fig. 15: Stretching rate $G_{s}$ versus normalized Lagrange parameter $\theta / 2 \pi(-\pi \leq \theta \leq \pi)$ at $k v_{\text {lin }} t=$ (a) 1.5 and (b) 4.0 , where $R_{A}^{2}=10^{3}$.

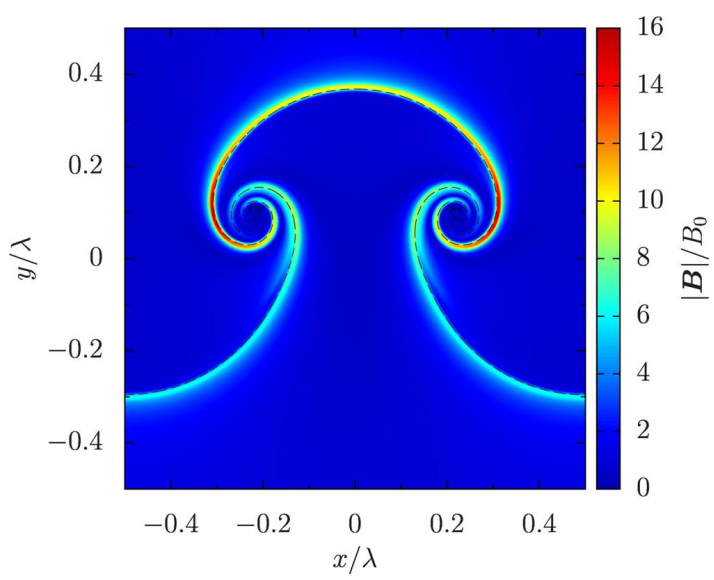

Fig. 16: Normalized magnetic field $|\boldsymbol{B}| / B_{0}$ at $k v_{l i n} t=4.0$ obtained by the ideal MHD simulation of a potential flow, where $A=-0.2$ and $R_{A}^{2}=10^{3}$. 
early time, the maximum stretching rate of the interface occurs at the top of the spike for both Atwood numbers due to the spike growth. However, at the later time shown in figure 15 (b), the large stretching rates are observed near $\theta /(2 \pi) \sim \pm 0.2$ for the case of $A=0$. This temporal change of the position from the spike to the vortex cores affects the growth rate of the magnetic field and causes a kind of inflection point on the growth of the magnetic field as shown in figure 13 (b) and (c). Since the large stretching of the interface occurs around the outside of the mushroom, the large magnetic field appears there as shown in figure 16, which is obtained from the corresponding ideal MHD simulation.

For all of the cases that the maximum magnetic field increases monotonically, the maximum magnetic field saturates at certain time. The maximum values of $R_{A}^{-2}\left|\boldsymbol{B} / \boldsymbol{B}_{0}\right|^{2}$ for $1 \leq R_{A}^{2} \leq 10^{3}$ in figure 13 attain an almost same value of $O(1)$ independent of $A$ and $R_{A}$, at which the saturation energy of the magnetic field coincides with the kinetic energy of RMI flows because

$$
\frac{1}{R_{A}^{2}}\left|\frac{\boldsymbol{B}_{\max }}{\boldsymbol{B}_{0}}\right|^{2}=\frac{2 B_{\max }^{2}}{\left[\left(\rho_{1}+\rho_{2}\right) v_{l i n}^{2}\right]} \equiv\left(\frac{v_{a}}{v_{l i n}}\right)^{2}=O(1) .
$$

From this estimate, we see that the magnetic field is amplified up to $|\boldsymbol{B}|>R_{A}$. A similar saturation of the amplified magnetic field is also observed in the ideal MHD simulations as shown in figures 6 and 7 , and the saturation level agrees with the one in figure 13.

The period of Alfvén oscillation observed for $R_{A}^{2}<10$ in figures 13 and 7 becomes smaller than that of the linear one because of the magnetic field amplification. For that case, the linear frequency $\omega$ in (3.5) should be replaced by the nonlinear frequency $\omega_{N L}=1 / R_{A}^{N L}$, where $R_{A}^{N L}$ is estimated as $R_{A}^{N L} \sim \gamma / B$.

\subsection{Surface Alfvén wave and MHD Richtmyer-Meshkov instability}

In previous subsections, we consider the nonlinear evolution of the interface only for the initial conditions of the surface current $j_{s}(\theta, t)=0$ and the velocity shear $\gamma(\theta, t) \neq 0$. In this subsection, we discuss the cases with the initial condition of $j_{s}(\theta, t) \neq 0$ and show the appearance of the surface Alfvén wave (eigenmode in the linear analysis in section 3) with the initial condition of $\gamma(\theta, t)=0$ and the unstable mode leading to MHD RMI with that of $\gamma(\theta, t) \neq 0$ and relatively large $R_{A}^{2}=10^{3}$. The linear solution (3.6) includes the mode that the initial surface current density $\boldsymbol{j}_{s} \neq 0$ and our model allows such solutions as long as the current free condition in the bulk is satisfied. Here, we present two examples of the initial condition $\boldsymbol{j}_{s} \neq 0$ and show that one of which leads to the surface Alfvén wave and the other MHD RMI.

The initial condition for the first case is given by

$$
\begin{aligned}
\eta & =\epsilon \cos x, \\
\gamma & =0, \\
\boldsymbol{j}_{s} & =(-2 \epsilon \cos x, 0),
\end{aligned}
$$

where $\epsilon$ is selected as $\epsilon=\sqrt{0.1}$ and $A=-0.2$. We show the temporal evolution of various physical quantities at a fixed Lagrange parameter $\theta=0$ or $\pi / 2$ for the surface Alfvén wave in (a) of figure 17 , where the normal velocity $v_{n}$ is defined as $v_{n}=\boldsymbol{q} \cdot \boldsymbol{n}$. Since the initial $\gamma=0$, the condition (6.3) reveals only the pure surface Alfvén wave. Figure (b) denotes the temporal change of the interfacial profile in this surface Alfvén wave solution. As shown in these two figures, all of the 



Fig. 17: Temporal evolution of physical quantities in (a) the surface Alfvén wave and (c) MHD RMI, and the interface structure in (b) the surface Alfvén wave at several $k v_{l i n} t$ and (d) MHD RMI at $k v_{l i n} t=4.0$. The vortex sheet strength $\gamma$, normal velocity $v_{n}$, the $y$ component of the magnetic field $\boldsymbol{B}_{2} ; B_{2 y}$, and the current sheet strength $j_{s}$ in (a) and (c) are selected at the Lagrange parameter $\theta=\pi / 2,0, \pi / 2$, and 0 , respectively. The color bar in (d) denotes the magnetic field $B_{2}^{t}$. Here, the Alfvén number $R_{A}$ in (a) and (b) [(c) and (d)] is selected as $R_{A}^{2}=0.1\left[R_{A}^{2}=10^{3}\right]$, and $A=-0.2$ for all figures. 
variables including the vortex sheet strength, the magnetic field, the current sheet strength, and the normal velocity oscillate with the Alfvén frequency given by equation (3.4) according to the normal mode (3.6). Namely, the physical quantities measured by $k v_{a 0} t$ oscillate with period $2 \pi$. The temporal evolution of the maximum value of the normalized magnetic field $|\boldsymbol{B}|^{2} /\left(R_{A}^{2} B_{0}\right)^{2}$ is almost same as the curve $R_{A}^{2}=0.1$ in figure 13 (b); i.e., the magnetic field amplification does not occur for this initial condition. It should be noted that Wheatley et al. found an oscillatory motion in MHD RMI when $R_{A} \ll 1$ (Wheatley et al., 2014). Therefore, their results might correspond to a surface Alfvén wave instead of MHD RMI.

The initial condition in (c) and (d) of figure 17 is given as

$$
\begin{aligned}
\eta & =\epsilon R_{A} \cos x, \\
\gamma & =-2 \sin x, \\
\boldsymbol{j}_{s} & =\left(-2 \epsilon R_{A} \cos x, 0\right),
\end{aligned}
$$

where the initial amplitude $\epsilon R_{A}$ is selected as $\epsilon R_{A}=0.1\left(R_{A}^{2}=10^{3}\right)$. The condition (6.4) is not the eigenmode of the linear solution (3.6). For this large velocity shear of $R_{A}^{2}=10^{3}$, the Alfvén oscillation does not occur; instead, an unstable mode leading to MHD RMI develops. All physical quantities in figure (c) (except the spike velocity $v_{n}$ ) grow in time and the roll-up of the interface (d) is the same as those in figure 9 in subsection 6.2. The magnetic field $\boldsymbol{B}_{2}$ is amplified up to the same degree as the one in figure 9 .

For the initial condition as the same given by equation (6.4) with $\epsilon R_{A}=0.1$ and $R_{A}^{2}=O(1)$, the oscillation of all physical quantities were observed, as similar as the solutions observed in figure 13 for $R_{A}^{2}=0.1$ and 1.0. However, it should be mentioned that their spatial profiles deviate from pure sinusoidal ones because of the velocity shear at the interface. For the parameter range of $R_{A}^{2}=O(1)$ with non-zero (non-uniform) velocity shear, the dynamics does not obey the linear RMI nor the linear surface Alfvén wave and thus the linear analysis is invalid. Our model is effective even in this nonlinear region.

\section{Conclusions}

We have developed a theoretical model to describe the fully nonlinear evolution of non-uniform current-vortex sheets under the assumption that the magnetic field is initially parallel to the sheet. The model consists of an equation for the sheet velocity and mutual coupling equations between the vortex sheet strength and the magnetic field on the sheet. It is shown that two-dimensional MHD shear flow problems are described only by one-dimensional Lagrange parameter on the sheet. In our model, when the initial conditions $\boldsymbol{\gamma}=\boldsymbol{u}_{1}-\boldsymbol{u}_{2}$ and $\boldsymbol{j}_{s}=\boldsymbol{B}_{1}-\boldsymbol{B}_{2}$ at the interface, and $\boldsymbol{j}=0$ and $\boldsymbol{\omega}=0$ in the bulk are provided with the boundary condition $\boldsymbol{B}=B_{0} \hat{\boldsymbol{e}_{x}}$ at $y= \pm \infty$, the interfacial dynamics, and the bulk velocity and magnetic fields are uniquely determined, no matter how extensively the interface deforms.

The linear eigenmode of the system is shown to be nothing but the surface Alfvén mode with density stratification. As an extension of our model, we have succeeded to calculate the magnetic field and fluid velocity in the bulk from only the quantities on the interface. Our model can be applied to general nonlinear interfacial motion and shear flows in MHD. Two-dimensional ideal MHD simulations show that the nonlinear dynamics of a shocked interface with density 
stratification (MHD RMI) agrees fairly well with that for its corresponding potential flow. By applying the model to MHD RMI, nonlinear amplification and saturation of magnetic field, and suppression of roll-up of the interface due to the magnetic tension have been obtained, which are found to be consistent with corresponding ideal MHD simulations.

Nonlinear evolution of the interface is shown to be determined by two parameters, the Alfvén and Atwood numbers. Their dependence on the nonlinear dynamics of the sheet and thus the magnetic field amplification are investigated for various initial conditions. It is shown by the model that the magnetic field amplification occurs locally depending on the nonlinear dynamics of the current-vortex sheet, which is also determined by the two parameters. The model calculations show that the magnetic field amplification is large enough to explain the long-standing paradigm of cosmic-ray proton acceleration in young SNRs (Sano et al., 2012). The sheet model can also capture various geometrical quantities such as curvature and stretching rate of the interface, which are hard to calculate by the ideal MHD simulations. Our model can be applied to general nonlinear interfacial motion in MHD flows not only in MHD RMI but MHD KHI, MHD RTI, and the surface Alfvén wave as well, if we change the initial velocity shear and/or take into account the gravity.

The current-vortex sheet does not persist as a steady solution if the magnetic field has a component normal to the interface. This is because the Alfvén wave can transport the vortex sheet away from the interface (Wheatley et al., 2009, Sano et al., 2013). The sheet model and the ideal MHD simulations play a complementary role. By utilizing these two, the understanding of MHD shear flows would be more advanced.

\section{Acknowledgement}

This work was supported by a Grant-in-Aid for Scientific Research (B) (Grant No. 26287147) and (C) (Grant No. 23540453) from the Japan Society for the Promotion of Science, a Grant-in-Aid for Research Promotion, Ehime university, and joint research project of ILE, Osaka University. The authors would like to thank Professor K. Hiraide and Professor S. Yanagi for their mathematical advice. We are deeply grateful to Professor A. Kageyama, Professor K. Kusano, Professor J. G. Wouchuk, and Professor Z. Yoshida for their valuable comments and discussions on plasma physics. We are also particularly indebted to Professor Y. Kaneda for his advice and suggestions.

\section{A Current density and surface current density}

Here, we mention the relation between the current density $\boldsymbol{j}$ and the surface current density $\boldsymbol{j}_{s}$ (or the current sheet strength $j_{s}$ ). From the integral form of the Ampère's law (2.5), the following relation holds for the (total) current $I$ :

$$
I \equiv \iint \boldsymbol{j} \cdot d \boldsymbol{A}=\iint(\nabla \times \boldsymbol{B}) \cdot d \boldsymbol{A}=\int\left(\boldsymbol{B}_{1}-\boldsymbol{B}_{2}\right) \cdot \boldsymbol{t} d s
$$

where $\boldsymbol{j}=j \hat{\boldsymbol{e}}_{z}, d \boldsymbol{A}=\hat{\boldsymbol{e}}_{z} d A$ is the area vector associated with a small rectangular area $d A=d \xi d s$ ( $d \xi$ is a small element in the direction of the unit normal $\boldsymbol{n}$ ) encircling the interface and $d s$ is the line element of the interface. The current sheet strength $j_{s} \equiv \int j d \xi$ is given by

$$
j_{s}=\frac{d I}{d s}=\left(\boldsymbol{B}_{1}-\boldsymbol{B}_{2}\right) \cdot \boldsymbol{t}
$$


From this equation, we obtain the relation

$$
\boldsymbol{B}_{1}-\boldsymbol{B}_{2}=j_{s} \boldsymbol{t}=j_{s} \boldsymbol{n} \times \hat{\boldsymbol{e}}_{z}=\boldsymbol{n} \times \int \boldsymbol{j} d \xi=\boldsymbol{j}_{s} .
$$

\section{B Velocity field in bulk}

Now we decompose the velocity field $\boldsymbol{u}$ as

$$
\boldsymbol{u}=\boldsymbol{u}_{s}+\nabla \Xi
$$

where $\boldsymbol{u}_{s}$ denotes a solenoidal vector field and $\Xi$ is a irrotational potential field that satisfies the Laplace equation $\triangle \Xi=0$ in the bulk and the boundary conditions:

$$
\begin{aligned}
& \triangle \Xi=0 \quad \text { in } D, \quad \Xi \rightarrow 0 \text { as } \boldsymbol{x} \rightarrow \infty \\
& \nabla \Xi \cdot \boldsymbol{n} \equiv \frac{\partial \Xi}{\partial n}=\boldsymbol{u} \cdot \boldsymbol{n}-\boldsymbol{u}_{s} \cdot \boldsymbol{n} \equiv U_{n}-q_{n} \text { on } \partial D .
\end{aligned}
$$

Since the normal component of the fluid velocity is continuous at the interface; $U_{n}=q_{n}$, we have

$$
\frac{\partial \Xi}{\partial n}=0 \text { on } \partial D
$$

The potential field that satisfies the conditions (B.1) and (B.3) is $\Xi=0$ only. Therefore,

$$
\boldsymbol{u}=\boldsymbol{u}_{s}
$$

holds in the velocity field. This solenoidal field corresponds to the vortex induced velocity $\boldsymbol{q}$ or $q^{*}$ in (2.13). Then the Birkhoff-Rott equation (2.13) can describe the bulk velocity field $\boldsymbol{u}=(u, v)$ in its complex form as

$$
u^{*}(z)=u-i v=\frac{1}{2 \pi i} \mathrm{P} . \mathrm{V} . \int_{-\infty}^{\infty} \frac{\gamma\left(\theta^{\prime}\right) s_{\theta}\left(\theta^{\prime}\right) d \theta^{\prime}}{z-Z\left(\theta^{\prime}\right)} \quad(z \in D) .
$$

We present this bulk velocity field at $t=4.0$ for the Atwood number $A=-0.2$ and the Alfvén (Mach) number $R_{A}^{2}=10^{3}$ in figure 8 (right). Unlike the magnetic field, we see that the velocity field has the normal component at the interface.

\section{Numerical methods for the computation of the sheet model}

In this appendix, we present numerical methods in order to solve the governing equations in the sheet model (2.12), (2.29), and (6.2) (Matsuoka and Nishihara, 2006). Discretized equations to Eq. (6.2) are given as

$$
\begin{aligned}
U_{j} & =-\frac{h}{4 \pi} \sum_{\substack{m=0 \\
m \neq j}}^{N-1} \frac{\sinh \left(Y_{j}-Y_{m}\right) \gamma_{m} s_{\theta, m}}{\cosh \left(Y_{j}-Y_{m}\right)-\cos \left(X_{j}-X_{m}\right)+\delta^{2}}, \\
V_{j} & =\frac{h}{4 \pi} \sum_{\substack{m=0 \\
m \neq j}}^{N-1} \frac{\sin \left(X_{j}-X_{m}\right) \gamma_{m} s_{\theta, m}}{\cosh \left(Y_{j}-Y_{m}\right)-\cos \left(X_{j}-X_{m}\right)+\delta^{2}},
\end{aligned}
$$


in which $X_{j} \equiv X\left(\theta_{j}\right), Y_{j} \equiv Y\left(\theta_{j}\right)$, and $\gamma_{j} \equiv \gamma\left(\theta_{j}\right)$ are expanded into discrete Fourier series

$$
\begin{aligned}
X_{j} & =\theta_{j}+\sum_{m=-M}^{M} \hat{X}_{m} \mathrm{e}^{i m \theta_{j}}, \\
Y_{j} & =\sum_{m=-M}^{M} \hat{Y}_{m} \mathrm{e}^{i m \theta_{j}}, \\
\gamma_{j} & =\sum_{m=-M}^{M} \hat{\gamma}_{m} \mathrm{e}^{i m \theta_{j}} \quad(j=0, \ldots N-1),
\end{aligned}
$$

with the derivatives

$$
\begin{aligned}
X_{\theta, m} & =1+\sum_{m=-M}^{M} i m \hat{X}_{m} \mathrm{e}^{i m \theta_{j}}, \\
Y_{\theta, m} & =\sum_{m=-M}^{M} i m \hat{Y}_{m} \mathrm{e}^{i m \theta_{j}} \\
\gamma_{\theta, m} & =\sum_{m=-M}^{M} i m \hat{\gamma}_{m} \mathrm{e}^{i m \theta_{j}}
\end{aligned}
$$

where $X_{\theta, m}=(\partial X / \partial \theta)_{m}$ and so on. Note that the derivatives (C.3) do not involve errors which necessarily arise in derivative representations by usual difference approximations.

As pointed out by Kerr (Kerr, 1988), point vortices, i.e., the grid points on the interface tend to make a cluster around a bubble when the Atwood number is high, which is caused by the fact that the velocity difference between a bubble and spike becomes large for higher Atwood numbers. As a result of that, grid points around a spike decrease, and the calculation fails due to numerical instabilities. In order to avoid that, we use a grid redistribution method developed by Baker et al. (Baker and Nachbin, 1998) so that grid points are arranged equidistantly. This method is analogous to the node spreading presented by Kerr so as to obtain equally located grid points (Kerr, 1988); however, the accuracy of the grid redistribution method is higher than his method, and we can take more grid points. The grid redistribution method is as follows. Now we have a representation for the interface $(X(\theta, t), Y(\theta, t))$ with equally spaced Lagrangian markers $\theta$ at a time $t$. Then we seek a new mapping from $[0,2 \pi]$ onto itself, $\theta \rightarrow p$, such that

$$
p=\frac{1}{L} \int_{0}^{\theta} s_{\theta}\left(\theta^{\prime}\right) d \theta^{\prime}
$$

in which $s_{\theta}=\sqrt{X_{\theta}^{2}+Y_{\theta}^{2}}$ and $L$ is the whole length of the interface at the time $t$ :

$$
L=\frac{1}{2 \pi} \int_{0}^{2 \pi} s_{\theta}\left(\theta^{\prime}\right) d \theta^{\prime}
$$

Since we want to evenly spaced grid points $p=m h(m=0, \ldots N)$, where $h=2 \pi / N, N$, the number of grid points, we seek the following sequence:

$$
m h=\frac{1}{L} \int_{0}^{\bar{\theta}_{m}} s_{\theta}\left(\theta^{\prime}\right) d \theta^{\prime}, \quad(m=0, \ldots N)
$$




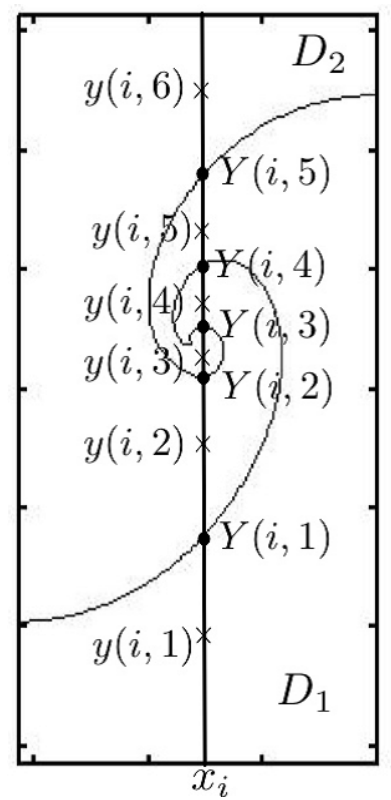

Fig. 18: Schematic figure of the interface, grid points $y(i, j)$, and the crossing points $Y(i, k)$ $(k=1,2 \cdots K)$ for a fixed $x_{i}$, where the cross and the black circle denote $y(i, j)$ and $Y(i, k)$, respectively. In this example, we set $K=5$.

Note that $\bar{\theta}_{m}(m=0, \ldots N)$ with $\bar{\theta}_{0}=\theta_{0}$ and $\bar{\theta}_{N}=\theta_{N}$ in equation (C.6) mapped to $m h$ in $\mathrm{p}$ is not equally divided $\theta_{m}=2 \pi m / N$ but a new position in $\theta$ which are not necessarily evenly spaced. In order to find these new parameterizations successively, Newton's method is used. Integrals in equations (C.4) and (C.5) are evaluated by the Fourier series of the integrand. Once new marker $\bar{\theta}_{m}$ is given, evenly spaced new position $\left(X\left(\bar{\theta}_{m}, t\right), Y\left(\bar{\theta}_{m}, t\right)\right)$ and the strength $\gamma\left(\bar{\theta}_{m}, t\right)$ are determined by cubic splines using $\theta_{m}, \bar{\theta}_{m},\left(X\left(\theta_{m}, t\right), Y\left(\theta_{m}, t\right)\right)$, and $\gamma\left(\theta_{m}, t\right)$. Thus, the redistribution of grid points at a time $t$ is completed. With these new dependent variables, new velocities $X_{t}\left(\bar{\theta}_{m}, t\right)$, $Y_{t}\left(\bar{\theta}_{m}, t\right)$, and $\gamma_{t}\left(\bar{\theta}_{m}, t\right)$ are evaluated at time $t$, then we can regard the discrete variable $\theta_{j}$ in the Fourier series $X_{j}-\bar{\theta}_{j}, Y_{j}, \gamma_{j}$ as the ones in the mapped space $p$, where the points are distributed with equal interval $h$. This redistribution is performed every time step.

\section{Evaluation of mesh points for a multi-valued interface}

In order to numerically calculate the bulk magnetic field for an interface that is multi-valued as shown in figure 9 , we need to distinguish that a grid point $\boldsymbol{x}_{i}=\left(x_{i}, y_{i}\right)(i \in \mathbb{N})$ in the plane is on which side (above or below) of the interface. In this appendix, we present a method of evaluation for that. Suppose that $y(i, j)(i, j \in \mathbb{N})$ denotes the $y$ coordinate designated by a mesh point $(i, j)$ ( $i$ : fixed) and $Y(i, k)\left(k \in \mathbb{N}, i\right.$ : fixed) is the $y$ coordinate that the line $x_{i}=$ constant and the interface intersect, where the integer $k$ satisfies $k \leq K, K$ the number of intersection points (refer to figure 18).

When the point $\boldsymbol{x}_{i}$ is located below of the interface $\left(\boldsymbol{x}_{i} \in D_{1}\right)$, the integer $k$ that satisfies $y(i, j)>Y(i, k)$ becomes even (e.g., the point $y(i, 5) \in D_{1}$ satisfies $y(i, 5)>Y(i, 4)(k=4)$ in 


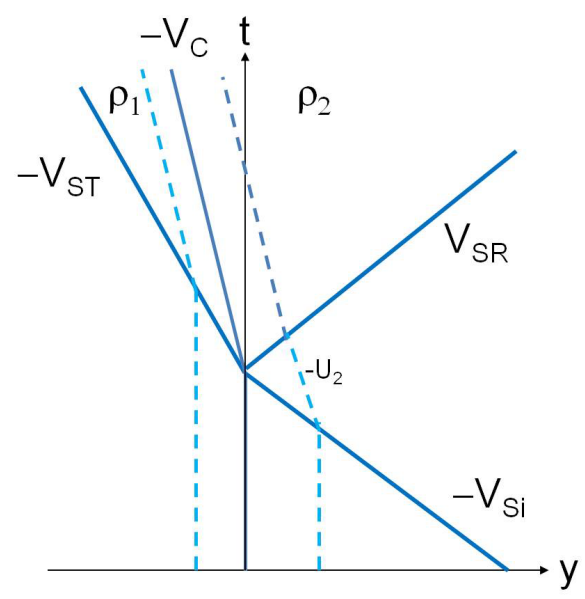

Fig. 19: Shock flow diagram for a reflected shock case in a laboratory frame. A incident shock propagates from the right to the left with shock speed of $-V_{S i}$, where $-U_{2}$ is the fluid velocity behind the incident shock, $V_{S R}$ and $-V_{S T}$ are the reflected and transmitted shock speeds, respectively, and $-V_{C}$ is the speed of the contact surface after the shock interaction. Here, $\rho_{1}$ and $\rho_{2}$ are the mass densities behind the transmitted and reflected shocks, respectively.

figure 18). On the other hand, when the point $\boldsymbol{x}_{i}$ is located above of the interface $\left(\boldsymbol{x}_{i} \in D_{2}\right)$, the integer $k$ that satisfies $y(i, j)>Y(i, k)$ becomes odd (e.g., the point $y(i, 6) \in D_{2}$ satisfies $y(i, 6)>Y(i, 5)(k=5)$ in figure 18$)$.

In order to find the intersection points $Y(i, k)(k=1,2 \cdots K)$ numerically, we increase the number of grid point of the interface $N(N=512$ here) to $m N(m \in \mathbb{N})$ in advance by the interpolation (we adopt the spline interpolation here) and detect $Y(i, k)$ for a fixed $x_{i}$, where we select $m=20 \sim 60$. If the condition $|y(i, j)-Y(i, k)|<\epsilon(0<\epsilon \ll 1)$ is satisfied between a point on the interface $y(i, j)$ and an intersection point $Y(i, k)$, we regard the point $Y(i, k)$ as being located on the interface, where we select e.g., as $\epsilon=10^{-4}$.

\section{E Linear growth rate of Richtmyer-Meshkov instability}

We consider the same configuration as Richtmyer (Richtmyer, 1960) introduced as shown in figure 19 , where an incident shock propagates through a light fluid $(i=2)$ in the $-y$-direction with shock speed $-V_{S i}$ in the laboratory system of reference and fluid velocity behind the incident shock is $-U_{2}$. When it hits the contact surface between the light and heavy fluids, the reflected shock starts to move in the $y$-direction and the transmitted one does in the $-y$-direction with the shock speeds of $V_{S R}$ and $-V_{S T}$, respectively. The contact surface moves to the $-y$-direction with a speed of $-V_{C}$ and two fluids behind the reflected and transmitted shocks also move with the same velocity $-U=-V_{C}$. The mass density of the heavy fluid and that of light fluid after the shock interaction are denoted as $\rho_{1}$ and $\rho_{2}$, respectively.

We assume the initial ripple of the interface to be of the form $\psi_{0} \cos k x$. Hence, just after the shock refraction at $t=0+$, the reflected and transmitted shock fronts are deformed, and those of the initial ripples resemble that at the contact surface, but with different amplitudes. A simple 
kinematic calculation shows that the amplitudes of initial shock ripples are given by (Richtmyer, 1960)

$$
\psi_{R 0}=\left(1+\frac{V_{S R}}{V_{S i}}\right) \psi_{0} \quad \text { and } \quad \psi_{T 0}=\left(1-\frac{V_{S T}}{V_{S i}}\right) \psi_{0}
$$

for the reflected and transmitted fronts, respectively. As is well known, the tangential velocity must be continuous across any deformed shock fronts. For the geometry shown in figures 1 and 19 , the initial tangential velocities can be equal to

$$
\delta v_{1}^{*}=-U k \psi_{T 0} \quad \text { and } \quad \delta v_{2}^{*}=\left(U_{2}-U\right) k \psi_{R 0} .
$$

These two tangential velocities provide the initial circulation that is distributed along the contact surface ripple proportional to $\sin k x$, which is actually the initial cause of the growth of the contact surface ripple (Fraley, 1986, Samtaney and Zabusky, 1993, 1994, Velikovich, 1996, Wouchuk and Nishihara, 1997, Wouchuk and Cavada, 2004, Herrmann et al., 2008). As the shocks separate away from the interface, their corrugation amplitudes oscillate and decrease with time. As the shock ripples evolve in time, pressure perturbations are generated behind the shocks and propagate through the compressed fluids with the local sound speed. As a result, the interface ripple and the shock ripples interact with each other, and they oscillate in time and asymptotically tend to zero. For an ideal gas EOS, the pressure perturbation at the interface decreases asymptotically in time as $t^{-3 / 2}$ (Zaidel, 1960, Fraley, 1986, Wouchuk and Cavada, 2004) independent with the shock intensity. It should also be noted that the rippled shock leaves vorticity behind in the bulk, but they are the second order and also decays with time (Wouchuk and Nishihara, 1997). Therefore no baroclinic effects are required for RMI.

The asymptotic interface growth rate can be obtained within a linear theory and a weak shock limit by integrating tangential component of equation of motion

$$
\rho_{i} \frac{\partial \delta \tilde{v}_{x i}}{\partial t}=-k \delta \tilde{p}_{i}(t)
$$

where we assumed that $\delta p_{i}=\delta \tilde{p}_{i}(y, t) \cos k x$ and $\delta v_{x i}=\delta \tilde{v}_{x i}(y, t) \sin k x$. This equation is valid at both sides of the contact surface at any time $t>0+$. From the pressure continuity at $y=0$ and the integration in the time interval $0+<t<\infty$, we obtain a relation for the asymptotic tangential velocity $\delta \tilde{v}_{x i}^{\infty}$ in fluid $i=1$ or 2 as

$$
\rho_{1}\left(\delta \tilde{v}_{x 1}^{\infty}-\delta v_{1}^{*}\right)=\rho_{2}\left(\delta \tilde{v}_{x 2}^{\infty}-\delta v_{2}^{*}\right) .
$$

Since the normal component of fluid velocity is continuous at the interface and the velocity becomes irrotational asymptotically in a weak shock limit, the linear growth rate $v_{l i n}$ can be obtained from equation (E.4) as

$$
v_{\text {lin }}=\frac{\rho_{2} \delta v_{2}^{*}-\rho_{1} \delta v_{1}^{*}}{\rho_{1}+\rho_{2}} .
$$

It should be noted that the growth rate becomes smaller than the above growth rate for a strong shock because the bulk vorticity left by the ripple shocks suppresses the growth (Wouchuk and Nishihara, 1997). The pressure perturbation at the interface decays with time as $t^{-3 / 2}$, independent of the shock Mach number. As a consequence, the plasma velocity perturbations become incompressible and density perturbations approach zero for a very large time, as assumed in the present current-vortex sheet model. The potential flows assumed in the bulk; however, generate the velocity shear at the interface. 


\section{References}

Y. Agliskiy, A. Velikovich, M. Karasik, V. Serlin, C. Pawley, A. Schmitt, S. Obenschain, A. Mostovych, J. Gardner, and N. Metzler. Direct observation of mass oscillation due to ablative Richtmyer-Meshkov instability in plastic targets. Phys. Rev. Lett., 87:265001, 2001.

A. Alexakis. Large-scale magnetic fields in magnetohydrodynamic turbulence. Phys. Rev. Lett., 110:084502, 2013.

W. D. Arnett, J. N. Bahcall, R. P. Kirshner, and S. E. Woosley. Supernova 1987a. Ann. Rev. Astron. Astrophys., 27:629-700, 1989.

I. L. Arshukova, V. E. Nikolai, and H. K. Biernat. Magnetohydrodynamic instability of a high magnetic shear layer with a finite curvature radius. Phys. Plasmas, 9:401-408, 2002.

W. I. Axford. The stability of plane current-vortex sheets. Q. J. Mech. Appl. Math., 13:314-324, 1960.

W. I. Axford and C. O. Hines. A unifying theory of high-latitude geophysical phenomena and geomagnetic storms. Canadian J. Phys., 39:1433-1464, 1961.

G. Baker and A. Nachbin. Stable methods for vortex sheet motion in the presence of surface tension. SIAM J. Sci. Comput., 19:1737-1766, 1998.

G. Baker, D. I. Meiron, and S. A. Orszag. Generalized vortex methods for free surface flow problems. J. Fluid Mech., 123:477-501, 1982.

G. Birkhoff. Helmholtz and taylor instability. Proc. Symp. Appl. Maths. Soc., 13:55-76, 1962.

M. Brouillette. The Richtmyer-Meshkov instability. Ann. Rev. Fluid Mech., 34:445-468, 2002.

J. Cao, Z. Wu, H. Ren, and D. Li. Effects of shear flow and transverse magnetic field on RichtmyerMeshkov instability. Phys. Plasmas, 15:042102, 2008.

L. Chen and A. Hasegawa. A theory of long period magnetic pulsations, 2. impulse excitation of surface eigen-modes. J. Geophys Res., 79:1033-1037, 1974.

D. A. Clarke. A consistent method of characteristics for multidimensional magnetohydrodynamics. Astrophys. J., 457:291-320, 1996.

P. Colella and P. R. Woodward. The piecewise parabolic method (ppm) for gas-dynamical simulations. J. Comp. Phys., 54:174-201, 1984.

H. Daido, F. Miki, M. Fujita, K. Sawai, H. Fujita, Y. Kitagawa, S. Nakai, and C. Yamanaka. Generation of a strong magnetic field by an intense co2 laser pulse. Phys. Rev. Lett., 56:846$849,1986$.

C. R. Evans and J. F. Hawley. Simulation of magnetohydrodynamic flows - a constrained transport method. Astrophys. J., 332:659-677, 1988.

G. B. Field and J. Hutchins. A statistical model of interstellar clouds 2. effect of varying cloud cross-sections and velocities. Astrophys. J., 153:737-742, 1968. 
G. Fraley. Rayleigh-Taylor stability for a normal shock wave-density discontinuity interaction. Phys. Fluids, 29:376-387, 1986.

S. Fujioka, Z. Zhang, and et al. Kilotesla magnetic field due to a capacitor-coil target driven by high power laser. Sci. Rep., 3:1170, 2013.

R. Gerwin. Hydromagnetic surface waves in a conducting liquid surrounded by a compressible gas. Phys. Fluids, 10:2164-1365, 1967.

J. Giacalone and J. R. Jokipii. Magnetic field amplification by shocks in turbulent fluids. Astrophys. $J, 663: \mathrm{L} 41-\mathrm{L} 44,2007$.

C. Godreche and P. Manneville. Hydrodynamics and nonlinear instabilities. Cambridge University Press, 1998.

V. Goncharov. Theory of ablative Richtmyer-Meshkov instability. Phys. Rev. Lett., 82:2091-2094, 1999.

J. F. Hawley and N. J. Zabusky. Vortex paradigm for shock-accelerated density-stratified interfaces. Phys. Rev. Lett., 63:1241-1244, 1989.

M. Herrmann, P. Moin, and S. I. Abarzhi. Nonlinear evolution of the Richtmyer-Meshkov instability. J. Fluid Mech., 612:311-338, 2008.

D. D. Holm, M. Nitsche, and V. Putkaradze. Euler-alpha and vortex blob regularization of vortex filament and vortex sheet motion. J. Fluid Mech., 555:149-176, 2006.

J. K. Hunter and J. B. Thoo. On the weakly nonlinear Kelvin-Helmholtz instability of tangential discontinuities in mhd. J. Hyper. Diff. Eqs., 8:691-726, 2011.

O. A. Hurricane, D. A. Callahan, D. T. Casey, P. M. Celliers, C. Cerjan, E. L. Dewald, T. R. Dittrich, T. Döppner, D. E. Hinkel, L. F. Berzak Hopkins, J. L. Kline, S. Le Pape, T. Ma, A. G. MacPhee, J. L. Milovich, A. Pak, H.-S. Park, P. K. Patel, B. A. Remington, J. D. Salmonson, P. T. Springer, and R. Tommasini. Fuel gain exceeding unity in an inertially confined fusion implosion. Nature, 506:343-348, 2014.

K. L. Ilin, Y. L. Trakhinin, and V. A. Vladimirov. The stability of steady magnetohydrodynamic flows with current-vortex sheets. Phys. Plasmas, 10:2469-2658, 2003.

T. Inoue, J. Shimoda, Y. Ohira, and R. Yamazaki. The origin of radially aligned magnetic fields in young supewrnova remnants. Astrophys. J., 772:L20-24, 2012.

R. Ishizaki and K. Nishihara. Propagation of a ripple shock wave driven by nonuniform laser ablation. Phys. Rev. Lett., 78:1920-1923, 1997.

J. W. Jacobs and J. M. Sheeley. Experimental study of incompressible Richtmyer-Meshkov instability. Phys. Fluids, 8:405-415, 1996.

R. M. Kerr. Simulation of Rayleigh-Taylor flows using vortex blobs. J. Comput. Phys., 76:48-84, 1988. 
N. K-R. Kevkaham. The vorticity jump across a shock in a non-uniform flow. J. Fluid Mech., 341: 371-384, 1997.

H. Koyama and S. Inutake. An origin of supersonic motions in interstellar clouds. Astrophys, J., 564:L97-L101, 2002.

R. Krasny. A study of singularity formation in a vortex sheet by the point vortex approximation. J. Fluid Mech., 167:65-93, 1986.

R. Krasny. Computation of vortex sheet roll-up in the Trefftz plane. J. Fluid Mech., 184:123-155, 1987.

T. Ma, P. K. Patel, and et al. Onset of hydrodynamic mix in high-velocity, highly compressed inertial confinement fusion implosions. Phys. Rev. Lett., 111:085004, 2013.

C. Matsuoka. Three-dimensional vortex sheet motion with axial symmetry in incompressible Richtmyer-Meshkov and Rayleigh-Taylor instabilities. Phys. Scr., T155:014013, 2013.

C. Matsuoka and K. Nishihara. Vortex core dynamics and singularity formations in incompressible Richtmyer-Meshkov instability. Phys. Rev. E, 73, 74:026304, 049902(E), 2006.

C. Matsuoka, K. Nishihara, and Y. Fukuda. Nonlinear evolution of an interface in the RichtmyerMeshkov instability. Phys. Rev. E, 67, 68:036301, 029902(E), 2003.

E. E. Meshkov. Instability of the interface of two gases accelerated by a shock wave. Sov. Fluid Dynamics, 4:101-108, 1969.

W. Mostert, V. Wheatley, R. Samtaney, and D.I. Pullin. Effects of magnetic fields on magnetohydrodynamic cylindrical and spherical Richtmyer-Meshkov instability. Phys. Fluids, 27:104102, 2015.

W. C. Muller and R. Grappin. Spectral energy dynamics in magnetohydrodynamic turbulence. Phys. Rev. Lett., 95:114502, 2005.

K. Nishihara, J. G. Wouchuk, C. Matsuoka, R. Ishizaki, and V. V. Zhakhovskii. RichtmyerMeshkov instability: theory of linear and nonlinear evolution. Phil.Trans. Roy. Soc. A, 368: 1769-1807, 2010.

M. Nitsche and R. Krasny. A numerical study of vortex ring formation at the edge of a circular tube. J. Fluid Mech., 276:139-161, 1994.

L. J. Perkins, B. G. Logan, G. B. Zimmerman, and C. J. Werner. Two-dimensional simulations of thermonuclear burn in ignition-scale inertial confinement fusion targets under compressed axial magnetic fields. Phys. Plasmas, 20:072708, 2013.

D. I. Pullin. Numerical studies of surface-tension effects in nonlinear Kelvin-Helmholtz and Rayleigh-Taylor instability. J. Fluid Mech., 119:507-532, 1982.

R. D. Richtmyer. Taylor instability in shock acceleration of compressible fluids. Commun. Pure Appl. Math., 13:297-319, 1960. 
N. Rott. Diffraction of a weak shock with vortex generation. J. Fluid Mech., 1:111-128, 1956.

P. G. Saffman. Vortex dynamics. Cambridge University Press, 1992.

R. Samtaney. Suppression of the Richtmyer-Meshkov instability in the presence of a magnetic field. Phys. Fluids, 15:L53-L56, 2003.

R. Samtaney and N. J. Zabusky. On shock polar analysis and analytical expressions for vorticity deposition in shock-accelerated density-stratified interface. Phys. Fluids A, 5:1285-1287, 1993.

R. Samtaney and N. J. Zabusky. Circulation deposition on shock-accelerated plannar and curved density-stratified interfaces: models and scaling laws. J. Fluid Mech., 269:45-78, 1994.

T. Sano, S. Inutsuka, and S. M. Miyama. A saturation mechanism of magnetorotational instability due to ohmic dissipation . Astrophys. J, 506:L57-L60, 1998.

T. Sano, K. Nishihara, C. Matsuoka, and T. Inoue. Magnetic field amplification associated with the Richtmyer-Meshkov instability. Astrophys. J, 758:126, 2012.

T. Sano, T. Inoue, and K. Nishihara. Critical magnetic field strength for suppression of the Richtmyer-Meshkov instability in plasmas. Phys. Rev. Lett., 111:016102, 2013.

M. J. Shelley. A study of singularity formation in vortex-sheet motion by a spectrally accurate vortex method. J. Fluid Mech., 244:493-526, 1992.

S. N. Shore. Astrophysical hydrodynamics: an introduction, 2nd Ed. John Wiley \& Sons, 2007.

J. A. Stamper, E. A. McLean, and B. H. RIPIN. Studies of spontaneous magnetic field in laserproduced plasmas by faraday rotation. Phys. Rev. Lett., 40:1177-1181, 1978.

J. M. Stone and M. L. Norman. Zeus-2d: A radiation magnetohydrodynamics code for astrophysical flows in two space dimensions. ii. the magnetohydrodynamic algorithms and tests. Astrophys. J. Suppl., 80:791-818, 1992.

Y. Uchiyama, F. A. Aharonian, T. Tanaka, T. Takahashi, and Y. Maeda. Extremely fast acceleration of cosmic rays in a supernova remnant. Nature, 449:576-578, 2007.

B. van Leer. Towards the ultimate conservative difference scheme. v - a second-order sequel to godunov's method. J. Comp. Phys., 32:101-136, 1979.

A. L. Velikovich. Analytic theory of Richtmyer-Meshkov instability for the case of reflected rarefaction wave. Phys. Fluids, 8:1666-1679, 1996.

V. Wheatley, R. Samtaney, and D. I. Pullin. The Richtmyer-Meshkov instability in magnetohydrodynamics. Phys. Fluids, 21:082102, 2009.

V. Wheatley, R. Samtaney, D. I. Pullin, and R. M. Gehre. The transverse field Richtmyer-Meshkov instability in magnetohydrodynamics. Phys. Fluids, 26:016102, 2014.

J. G. Wouchuk. Growth rate of the linear Richtmyer-Meshkov instability when a shock is reflected. Phys. Rev. E, 63:056303, 2001. 
J. G. Wouchuk and J. L. Cavada. Spontaneous acoustic emission of a corrugated shock wave in the presence of a reflecting surface. Phys. Rev. E, 70:046303, 2004.

J. G. Wouchuk and K. Nishihara. Linear perturbation growth at a shocked interface. Phys. Plasmas, 3:3761-3776, 1996.

J. G. Wouchuk and K. Nishihara. Asymptotic growth in the linear Richtmyer-Meshkov instability. Phys. Plasmas, 4:1028-1038, 1997.

J. G. Wouchuk, Huete Ruiz de Lira, and A. L. Velikovich. Analytical linear theory for the interaction of a planar shock wave with an isotropic turbulent vorticity field. Phys. Rev. E, 79:066315, 2009 .

H. Yoneda, T. Namiki, and et al. Strong compression of a magnetic field with a laser-accelerated foil. Phys. Rev. Lett., 109:125004, 2012.

P. M. Zaidel. Shock wave from a slightly curved piston. J. Appl. Math. Mech., 24:316-327, 1960. 